\title{
Relating 27-day Averages of Solar, Interplanetary Medium Parameters and Geomagnetic Activity Proxies in Solar Cycle 24
}

\section{Yvelice Soraya Castillo ( $\nabla$ yvelice.castillo@unah.edu.hn )}

Universidad Nacional Autónoma de Honduras https://orcid.org/0000-0003-3119-6512

\section{Maria Alexandra Pais}

University of Coimbra: CITEUC, Geophysical and Astronomical Observatory

\section{João Fernandes}

University of Coimbra: CITEUC, Geophysical and Astronomical Observatory

\section{Paulo Ribeiro}

University of Coimbra: CITEUC, Geophysical and Astronomical Observatory

\section{Anna L. Morozova}

Hungarian Academy of Sciences Research Centre for Astronomy and Earth Sciences Geodetic and Geophysical Institute: Magyar Tudomanyos Akademia Csillagaszati es Foldtudomanyi Kutatokozpont Geodeziai es Geofizikai Intezet

\section{Fernando J. G. Pinheiro}

UC: Universidade de Coimbra

\section{Research Article}

Keywords: Solar Active Regions, Solar Wind Disturbances, Magnetosphere, Geomagnetic Disturbances, Bartel's rotation, Russell-McPherron mechanism, Tsygnanko and Sitnov model

Posted Date: March 6th, 2021

DOl: https://doi.org/10.21203/rs.3.rs-224356/v1

License: (c) (i) This work is licensed under a Creative Commons Attribution 4.0 International License. Read Full License

Version of Record: A version of this preprint was published at Solar Physics on July 27th, 2021. See the published version at https://doi.org/10.1007/s11207-021-01856-8. 


\title{
Relating 27-day Averages of Solar, Interplanetary Medium Parameters and Geomagnetic Activity Proxies in Solar Cycle 24
}

Solar Physics

Yvelice Castillo ${ }^{1,2,3}$ (1) $\cdot$ Maria

Alexandra Pais ${ }^{2,3}$ (1) - João Fernandes ${ }^{2,4}$ (1)

Paulo Ribeiro ${ }^{2}$ (I) Anna L. Morozova ${ }^{2}$ (D) -

Fernando J. G. Pinheiro' ${ }^{2}$ (i)

(C) Springer

\begin{abstract}
Correlations between solar, interplanetary medium parameters and geomagnetic activity proxies in 27-day averages (a Bartels' rotation) were analysed for the 2009-2016 time interval. In this analysis, two new proxies were considered: 1) $B_{Z S}$ GSM, calculated as the daily percentage of the IMF southward component along the GSM Z-axis and then averaged every 27 days; 2) four magnetospheric indices (T-indices), calculated from the local North-South
\end{abstract}

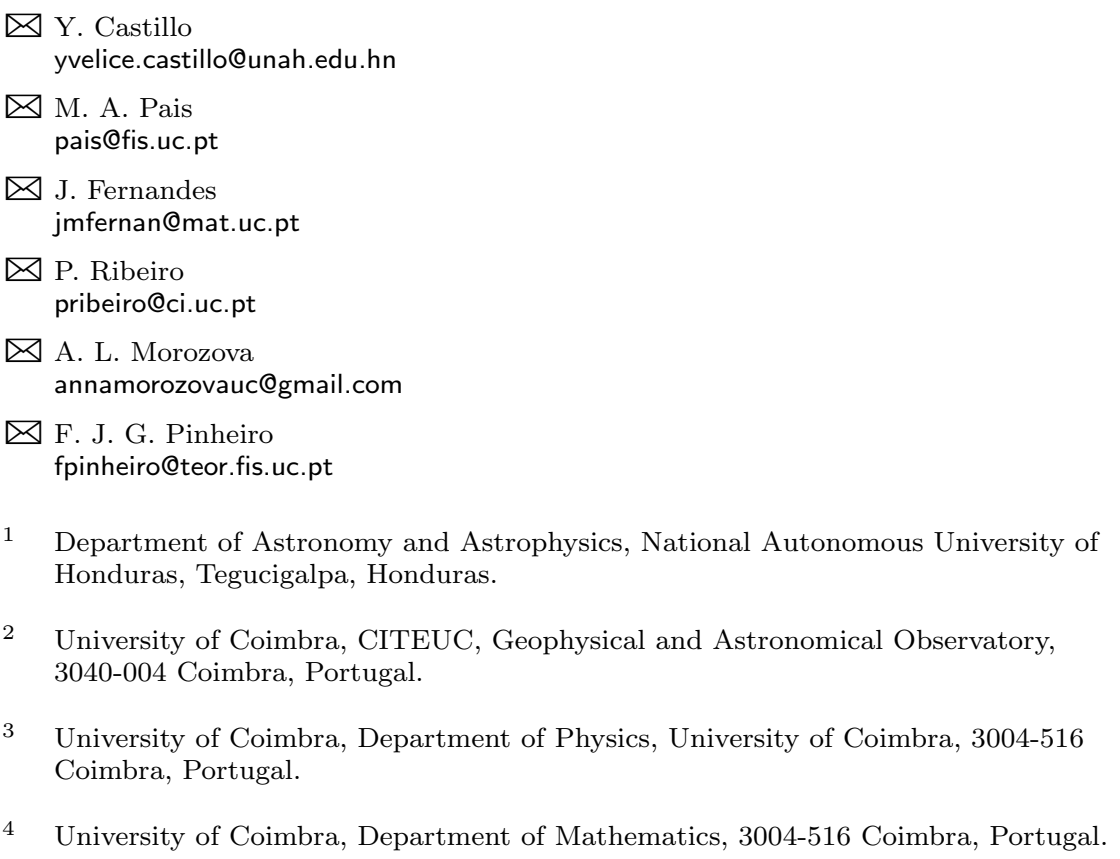


(X) contributions of the magnetosphere's cross-tail (TAIL), the symmetric ring current (SRC), the partial ring current (PRC) and the Birkeland current (FAC), derived from Tsyganenko and Sitnov 2005 (TS05) semi-empirical magnetospheric model. Our results suggest that, among the parameters tested in this study, solar facular areas, interplanetary magnetic field intensity and new proxies derived from TS05 model could be taken into account in an empirical model, with a 27-day resolution, to explain geomagnetic activity felt on the Earth's surface in terms of solar surface features and the IMF condition. We further retrieve a clear annual oscillation in series of 27-day-mean values of toward/away asymmetries of geomagnetic activity indices, which can be interpreted in the light of Russell-McPherron hypothesis for the semiannual variation of geomagnetic activity.

Keywords: Solar Active Regions; Solar Wind Disturbances; Magnetosphere; Geomagnetic Disturbances; Bartel's rotation; Russell-McPherron mechanism; Tsyganenko and Sitnov model.

\section{Overview}

Space Weather (SWE) concerns the variable conditions in the Sun and interplanetary medium that can have an impact on human safety and activities. This includes the electromagnetic perturbations due to solar and cosmic ray fluxes in the near-Earth environment and their effects in the technological and biological systems. The Sun and its activity is the main source of energy in the causal chain of processes of SWE. The most energetic solar events, such as white light flares and coronal mass ejections (CMEs), can drastically alter the level of electromagnetic and corpuscular radiation of interplanetary medium with consequent disturbance of the equilibrium state of the terrestrial magnetosphere-ionosphere coupled system, causing geomagnetic storms that can damage the navigation and communication systems and the ground power lines. SWE affects our modern way of life, sometimes in dramatic ways. Its potential impact is growing as we become more dependent of technological systems (Schrijver, 2015). The severity of the effects on almost all technologies depends in part on the geomagnetic latitude, being particularly important for the higher latitudes and for the South Atlantic Anomaly (Thomson, 2012). Mid-latitudes are also significantly perturbed by geomagnetic storms. These adverse effects include the loss of HF communications, damage in high-voltage power supply lines or pipelines and deterioration of services provided by global navigation satellite systems (e.g., Beggan et al., 2013; Buonsanto, 1999). A deeper understanding of SWE events can be used to forecast hazards and to define strategies for mitigating their impact. Interest in, and dependence on, SWE information and services grows rapidly, due to its importance for technological infrastructures, impact on global economy and growing need for SWE hazards mitigation (Schrijver, 2015).

The comparison between solar parameters, parameters of the solar wind and geomagnetic activity indices is of particular interest for identification of causal relationships between solar and terrestrial phenomena. The main goal of this 
study is to identify those solar, interplanetary medium and geomagnetic activity parameters that show the strongest statistical relations, and to sort out the best candidates to relate meaningfully the solar activity to geomagnetic disturbances felt on Earth's surface. We both use widely known proxies, like Sun's northern and southern facular areas (FA-N and FA-S), total and southward components of the interplanetary magnetic field ( $B$ and $B_{Z}$, respectively) or the Newell's coupling function (NLL), and test two new types of parameters. The first new parameter is the daily percentage of the southward component of the interplanetary magnetic field (IMF) in the Geocentric Solar Magnetospheric (GSM) coordinate system, which we call $B_{Z S}$. Another group of new proposed parameters consists in indices derived from simulations, using the TS05 (Tsyganenko and Sitnov, 2005) magnetospheric semi-empirical model of the geomagnetic field variations near the ground at middle latitudes, which we call T-indices.

\subsection{Solar hemispherical asymmetry}

Solar magnetic activity is controlled by differential rotation and meridional circulation in both hemispheres, but manifests differently in each one. Waldmeier (1971) found that the Sun's hemispheric asymmetry extends to different aspects of magnetic activity. During the last years the asymmetries in variations of many solar parameters have been confirmed by several authors: for the sunspot number and areas (Ballester, Oliver, and Carbonell, 2005; Temmer et al., 2006), facular region areas (Gonçalves et al., 2014), X-ray flares flux (Joshi et al., 2015), velocity of torsional oscillations (Lekshmi, Nandy, and Antia, 2018), and for the temporal delay on the reversal time polarity of the north and south Sun's magnetic field (Janardhan et al., 2018). It was also shown that those asymmetries might have an impact on the earth climate (Georgieva et al., 2007). A phase lag of activity in the northern and southern solar hemispheres typically ranges between one day to a year or two (Dorotovic et al., 2010). This suggests that the coupling between the two hemispheres is variable and weak (Norton and Gallagher, 2009). The north-south asymmetry of sunspot activity results in asynchronous reversal of the Sun's polar field and may affect the strength of the solar cycle. It appears that cycles with strong asymmetry tend to have a lower amplitude (e.g., cycles 23 and $24)$ in comparison with cycles in which sunspot activity in the two hemispheres is more synchronized (e.g., cycles 21 and 22) (Mordvinov et al., 2016). On the other hand, the causes of the asymmetry are on debate. Several authors point out a connection between the asymmetry and the solar dynamo (see Schüssler and Cameron (2018), Nepomnyashchikh et al. (2019) and references therein). It seems that the combination of dipolar mode and quadrupolar modes, with periods of about 22 years and between 13 and 15 years, respectively, can reproduce the observed patterns of the north and south asymmetry. The study of the hemispheric asymmetry has become a valuable tool to evaluate solar activity properties and in development of the dynamo theories. For example, in the recent work of Perri et al. (2018), the simulations of the solar wind, coupling solar dynamo and solar wind models, showed the clear impact of the hemispheric asymmetry on the solar wind properties (velocity and magnetic fields). 
Here, we consider the effect of solar hemispherical asymmetries of the sunspot numbers (e.g., Temmer, Veronig, and Hanslmeier, 2002) using data from SILSO data/image (Royal Observatory of Belgium, Brussels) and of the facular areas computed from images taken at the spectroheliograph of the Geophysical and Astronomical Observatory of the University of Coimbra (OGAUC). The hypothesis that these asymmetries might constrain the geomagnetic activity on Earth is tested.

\subsection{Simulations of magnetospheric contributions}

In Castillo et al. (2017), the TS05 model was tested against measurements at the Earth's surface, and shown to be a useful tool to help understanding the geomagnetic activity observed on the X-component. For the four mid-latitude stations considered in that study, and using in the analysis all geomagnetically active days during the 8-year period from 2007 to 2014, around 50\% of observed daily series were reproduced by TS05 model with correlation values above 0.7 . TS05 model estimates are computed using near-Earth satellite information of the solar wind plasma and the interplanetary magnetic field (e.g., Tsyganenko and Sitnov, 2005; Tsyganenko and Andreeva, 2015) from the NASA/GSFC's OMNI data set through OMNIWeb (https://omniweb.gsfc.nasa.gov/form/omni_min.html). Input data comprise: the interplanetary magnetic field (IMF) components $\left(B_{X}\right.$ GSM, $B_{Y}$ GSM, $B_{Z}$ GSM), the flow speed components ( $V_{X}$ GSE, $V_{Y}$ GSE, $V_{Z}$ GSE), the solar wind ram pressure, proton density and proton temperature, the SYM-H index and the Earth's geomagnetic dipole tilt angle. Semi-empirical relations between the magnetospheric and solar wind/IMF parameters are used to compute the magnetic field vectors associated to five magnetospheric current systems: 1) the symmetrical ring current (SRC); 2) the asymmetrical, or partial ring current (PRC); 3) the cross-tail sheet current (TAIL); 4) a system of two vertical currents called field-aligned currents or Birkeland currents (FAC), that connect ionosphere and magnetosphere in the polar regions and 5) a current flowing in the magnetospheric boundary called the magnetopause current or the Chapman-Ferraro current (CF).

Since, in the TS05 model, the inner ring current and the more distant tail current sheet form a current system circulating close and parallel to the geomagnetic equator, the variability associated to these currents is more clearly retrieved in the X-component of the geomagnetic field (Castillo et al., 2017). We computed estimates of hourly values for the $\mathrm{X}$ component at the four northern hemisphere mid-latitude geomagnetic observatories of Coimbra (Portugal), Panagurishte (Bulgary), Novosibirsk (Russia) and Boulder (USA) (see Castillo et al. (2017)), for the period 2009-2016, keeping separate contributions from different sources. Table 1 shows the geographic and geomagnetic coordinates of the four observatories. Geomagnetic coordinates were computed using the IGRF12 model for 2010.0. The Y component variability, being strongly dependent on the FAC current system because of its poloidal symmetry, is expected to be significantly affected by the unrealistic closure of FAC through the Earth's center in the TS05 model. Statistical results reported by Castillo et al. (2017) confirmed that TS05 model performs better in reproducing the $\mathrm{X}$ than the $\mathrm{Y}$ 
variability. For this reason, only TS05 simulations for the X-component were used to compute the T-indices.

The paper is organized as follows: Section 1 gives a background for the present study. Section 2 presents the analyzed parameters and the methods used to calculate them. Section 3 presents results of the correlation analysis and discusses them. The main conclusions are drawn in Section 4.

Table 1. List of stations and their geographic and geomagnetic coordinates.

\begin{tabular}{ccccccc}
\hline \multirow{2}{*}{ Station } & & \multicolumn{2}{c}{ Geographic Coord. } & & \multicolumn{2}{c}{ Geomagnetic Coord. } \\
\cline { 6 - 6 } \cline { 5 - 6 } \cline { 5 - 6 } \cline { 5 - 6 } COI & & Lat. $\left({ }^{\circ} \mathrm{N}\right)$ & Long. $\left({ }^{\circ} \mathrm{E}\right)$ & & Lat. $\left({ }^{\circ} \mathrm{N}\right)$ & Long. $\left({ }^{\circ} \mathrm{E}\right)$ \\
PAG & 40.2 & 351.6 & & 43.8 & 72.1 \\
NVS & & 42.5 & 24.2 & & 40.5 & 105.1 \\
BOU & 40.1 & 83.2 & & 45.4 & 156.0 \\
\hline
\end{tabular}

\section{Data}

All parameters analyzed in this study were averaged over the Bartel's period of solar rotation (i.e., a 27-day time interval), in order to filter out the recurrence tendency on geomagnetic activity associated with distinct influence of different solar sectors. Time averaging over a Bartels' rotation period allows to estimate the mean influence that the Sun may have on the Earth, taking into account the effect of all solar longitudes. Parameters are listed in Table 2 together with their source databases. The analyzed time interval covers an abnormally long period of weak solar activity with a minimum of the total solar irradiance in 2009, and both the rising and part of the declining phases of solar cycle 24. This solar cycle is particularly interesting due to the triple polarity reversal in the Sun's Northern Hemisphere, its smaller amplitude with respect to previous cycles and the long duration of its minimum (Janardhan et al., 2018; Mordvinov et al., 2016). Some of the analyzed series are shown in Figures 1 - 4 as a function of Bartel's rotation number.

In order to perform a cross correlation analysis, we grouped the parameters into five sets: solar parameters (SP), parameters of the solar wind (SWP), IMF components (IMF), geomagnetic activity indices (GAI) and indices obtained from TS05's simulations, or T-indices (TI). 
Table 2. List of 33 parameters analyzed and their data sources, separated by SP, SWP, IMF, GAI and TI. Explanations in the text.

\begin{tabular}{|c|c|c|}
\hline $\mathrm{N}^{\circ}$ & Solar Parameters (SP) & Source \\
\hline 1 & FA-T, total facular area ( $\%$ of solar disk). & \\
\hline 2 & FA-N, northern facular area ( $\%$ of solar disk). & Calculated by Barata et al. (2018). \\
\hline 3 & FA-S, southern facular area ( $\%$ of solar disk). & \\
\hline 4 & SN-T, total international sunspot number. & \\
\hline 5 & SN-N, Northern hemisphere's sunspot number. & WDC-SILSO, Royal Observatory of Belgium, Brussels. \\
\hline 6 & SN-S, Southern hemisphere's sunspot number. & \\
\hline 7 & FA-NS, FA-N minus FA-S (\%). & Obtained from items (2) and (3). \\
\hline 8 & SN-NS, SN-N minus SN-S. & Obtained from items (5) and (6). \\
\hline \multirow[t]{2}{*}{9} & F10, solar radio flux of $10.7 \mathrm{~cm}(\mathrm{sfu})$ & $\begin{array}{l}\text { NASA/GSFC's OMNI daily data (https://omniweb.gsfc.nasa.gov/form/dx1. } \\
\text { html and King and Papitashvili (2005). }\end{array}$ \\
\hline & Solar Wind (SWP) and IMF parameters & \\
\hline $10-13$ & $\begin{array}{l}T \text {, proton temperature }\left(10^{3} \mathrm{~K}\right), \rho \text {, proton density }\left(\mathrm{N} / \mathrm{cm}^{3}\right), V \text {, bulk flow } \\
\text { speed }(\mathrm{km} / \mathrm{s}), p \text {, flow pressure }(\mathrm{nPa}) \text {. }\end{array}$ & NASA/GSFC's OMNI daily data. \\
\hline $14-16$ & $B_{X}, B_{Y}$ GSE and $B_{Z}$ GSE, nT. & \\
\hline $17-18$ & $B_{Y}$ GSM and $B_{Z}$ GSM, nT. & NASA/GSFC's OMNI daily data. \\
\hline \multirow[t]{2}{*}{19} & $B$, magnitude of average field vector, $\mathrm{nT}$. & \\
\hline & Ground geomagnetic activity indices (GAI) & \\
\hline 20 & Newell's coupling function, NLL & $\begin{array}{l}\text { Tsyganenko's yearly data files for TA15 model. Tsyganenko's 5-min } \\
\text { Newell's data can be downloaded at http://geo.phys.spbu.ru/ } \sim \text { tsyganenko/ } \\
\text { TA_2016_RBF. }\end{array}$ \\
\hline \multirow[t]{2}{*}{$21-27$} & $\mathrm{Kp}^{*} 10, \mathrm{AE}, \mathrm{AU}, \mathrm{AL}, \mathrm{PCN}(\mathrm{mV} / \mathrm{m})$, Dst $(\mathrm{nT}), \mathrm{Ap}(\mathrm{nT})$ indices & NASA/GSFC's OMNI daily data. \\
\hline & New indices & \\
\hline $28-29$ & $B_{Z S}$ GSE and $B_{Z S}$ GSM. & Calculated by us with NASA/GSFC's OMNI hourly data. \\
\hline $30-33$ & T-TAIL, T-SRC, T-PRC and T-FAC indices (nT). & $\begin{array}{l}\text { Calculated by us from their respective TS05's sources. For more details } \\
\text { see Castillo et al. }(2017) \text {. }\end{array}$ \\
\hline
\end{tabular}




\subsection{Solar Parameters (SP)}

Most of the analyzed solar parameters refer to features at the solar photosphere and chromosphere: the international northern/southern hemisphere's sunspot number $(\mathrm{SN}-\mathrm{N} / \mathrm{SN}-\mathrm{S})$, the international total sunspot number $(\mathrm{SN}-\mathrm{T})$, the difference between northern and southern sunspot numbers (SN-NS), the northern/southern hemisphere's facular area (FA-N/FA-S), the difference between northern and southern facular areas (FA-NS) - see Fig. 1. The sunspot numbers are obtained from the SILSO (Sunspot Index and Long-term Solar Observations) database at the Royal Observatory of Belgium. The facular areas were calculated by Barata et al. (2018), using the computational tool developed by them. The 27-day-averaged data is shown at Figure 1 . We also used the solar radio flux at $10.7 \mathrm{~cm}$ wavelength (F10.7), originating in the chromosphere and corona of the Sun.

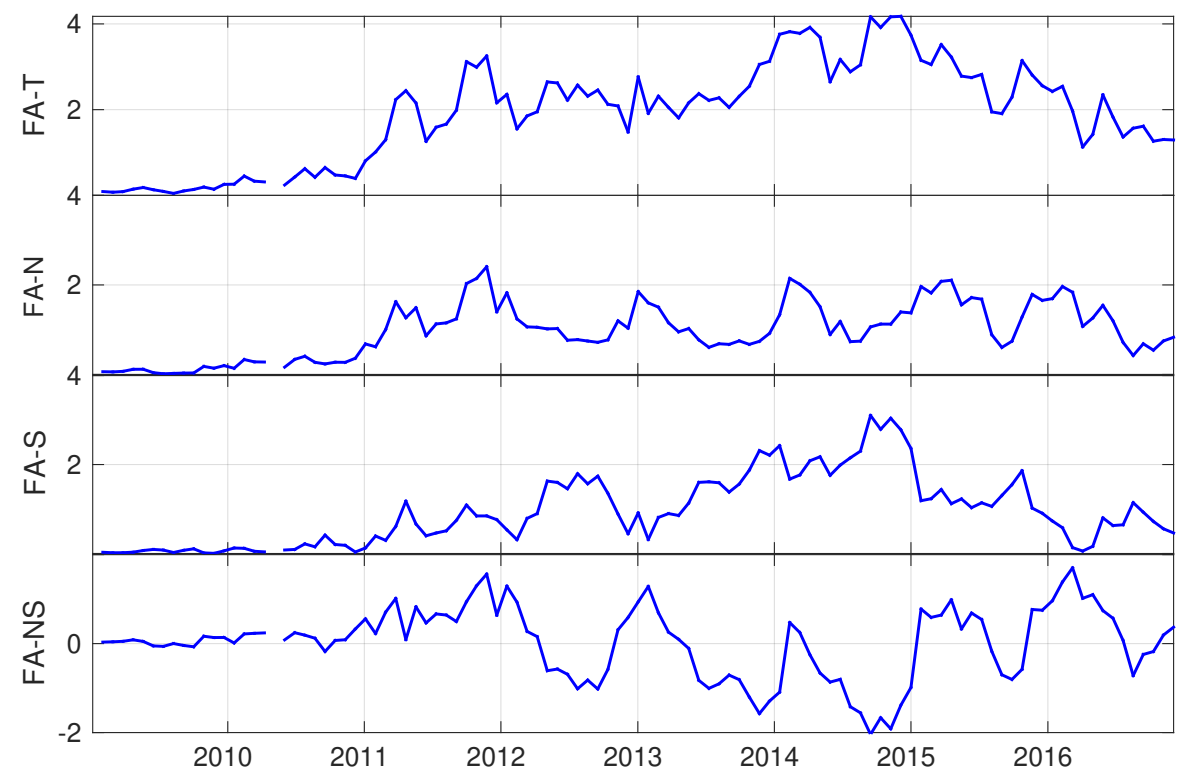

Figure 1. Time series of 27-day-averaged facular areas, in percentage of total solar disk area.

\subsection{Solar Wind (SWP) and IMF Parameters}

Solar wind parameters are the solar wind bulk speed in $\mathrm{km} / \mathrm{s}(V)$, proton flux temperature in Kelvin $(T)$, proton density in $\mathrm{N} / \mathrm{cm}^{3}(\rho)$, where $\mathrm{N}$ is the number of protons, and solar wind proton ram pressure in $\mathrm{nPa}(p)$, measured by spacecraft at the outer magnetosphere.

The IMF parameters are the components of the interplanetary magnetic field $B_{X}, B_{Y}, B_{Z}$, in $\mathrm{nT}$, in two different reference frames, both having the Xaxis pointing away from the Earth toward the Sun: the GSM and the GSE 
(Geocentric Solar Ecliptic). While GSM has the Y-axis always perpendicular to the geomagnetic dipole axis, GSE has the Y-axis in the ecliptic plane pointing toward dusk, independent of the geomagnetic dipole position (e.g., Laundal and Richmond, 2016). The total IMF field intensity, B, is also analyzed. Most of the data sets were obtained from the LRO NASA/GSFC's OMNI dataset. All 27day averages are calculated over the daily averages (see Figure 2). More details can be found at https://omniweb.sci.gsfc.nasa.gov/html/ow_data.html\#1.

\subsection{Ground Geomagnetic Activity indices (GAI) and Newell's Coupling Function (NLL)}

GAI indices used are PCN (polar cap north), AE, AU, AL, Dst, Kp*10 and Ap.

The Polar Cap North (PCN) 1-min index aims at characterizing the magnetic activity in the North polar cap that is driven by the IMF Bz component. It is deduced from the deviations in the horizontal $\mathrm{H}$ and $\mathrm{D}$ magnetic field components from the quiet level at the Qaanaaq (formerly known as Thule, in Greenland) polar cap station.

$\mathrm{AE}, \mathrm{AU}$ and AL 1-min indices monitor the magnetic signature of the auroral electrojets in the Northern hemisphere. The magnetograms of the horizontal components from the $12 \mathrm{AE}$ stations $\left(>56.5^{\circ} \mathrm{N}\right)$ are superimposed in plots against UT. The index AE at any epoch is defined by the separation between the upper $(\mathrm{AU})$ and lower $(\mathrm{AL})$ values at that epoch: $\mathrm{AE}=\mathrm{AU}-\mathrm{AL}$ (Davis and Sugiura, 1966). The AU and AL indices are intended to express the strongest current intensity of the eastward and westward auroral electrojets, respectively. The AE index represents the overall activity of the electrojets (Menvielle et al., 2011).

The disturbance field (Dst) is the storm-time decrease in H, measured at the Earth's surface as a result of geomagnetic activity. The Dst index is linearly dependent on the amplitude of the geomagnetic perturbation and is derived from hourly values of the horizontal geomagnetic component $(\mathrm{H})$ obtained at four magnetic observatories at low and mid-latitudes and distributed evenly in longitude: Honolulu (Hawaii), San Juan (Puerto Rico), Hermanus (South Africa) and Kakioka (Japan) (see ??).

The K-index is related quasi-logarithmically to the geomagnetic disturbance amplitude measured for the horizontal component at a particular observatory, during a three-hour interval and after the quiet daily variation has been removed. The planetary $\mathrm{K}$ index, $\mathrm{Kp}$, is the weighted average of the local standardized "K" indices of 13 geomagnetic observatories between $44^{\circ}$ and $63^{\circ}$ northern and southern geomagnetic latitudes. The 3 -hour $\mathrm{Kp}$ index ranges in a scale from 0 (quiet) to 9 (greatly disturbed). The $\mathrm{Kp}^{*} 10$ is similar to $\mathrm{Kp}$, with the difference that in the low resolution OMNI (LRO) data set the $\mathrm{Kp}^{*} 10$ average is rounded to its nearest integer (i. e., 10, 13, 17, 20, ...).

To calculate the daily average level of geomagnetic activity the Kp-scale must be converted back into an equivalent linear three hourly scale called ap-index, dividing by 2 the amplitude range corresponding to each Kp. The Ap-index is calculated as the arithmetic average of eight 3-hourly averaged ap-indices of 


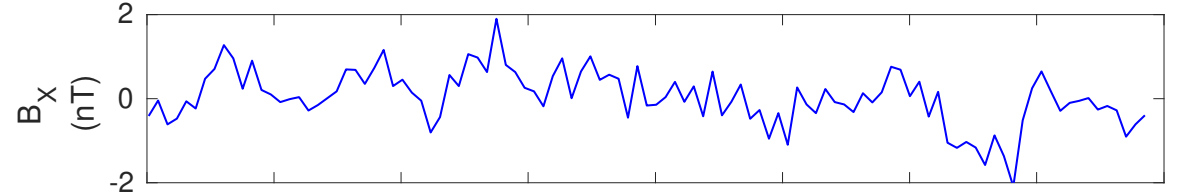

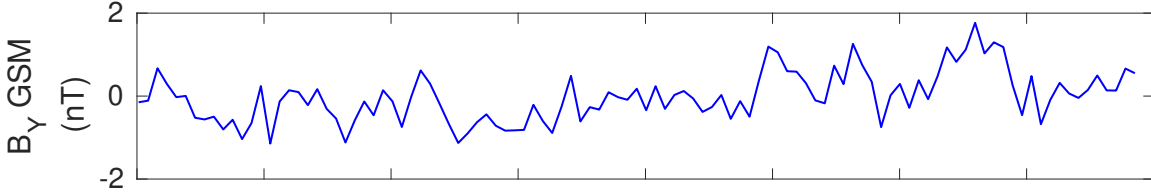

$\sum_{\infty}^{N}$

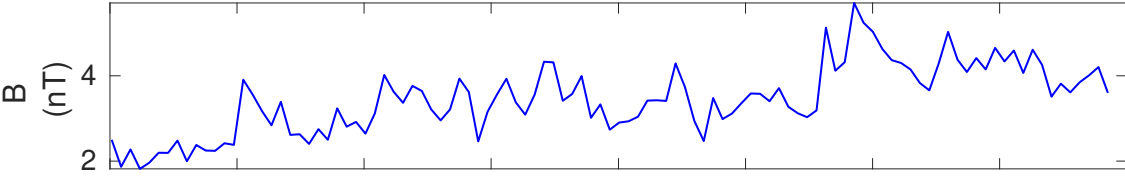

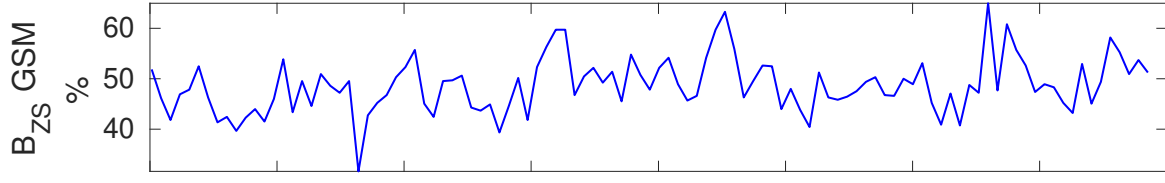

政

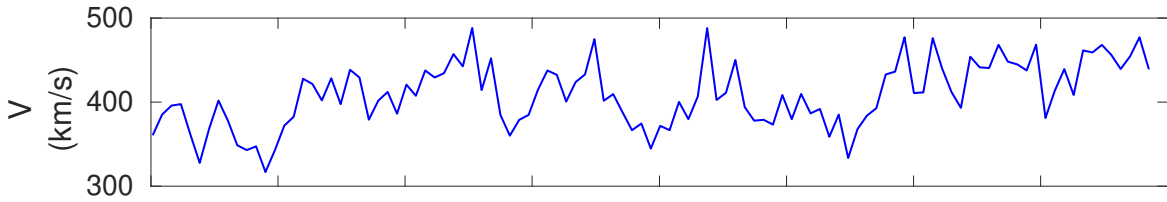

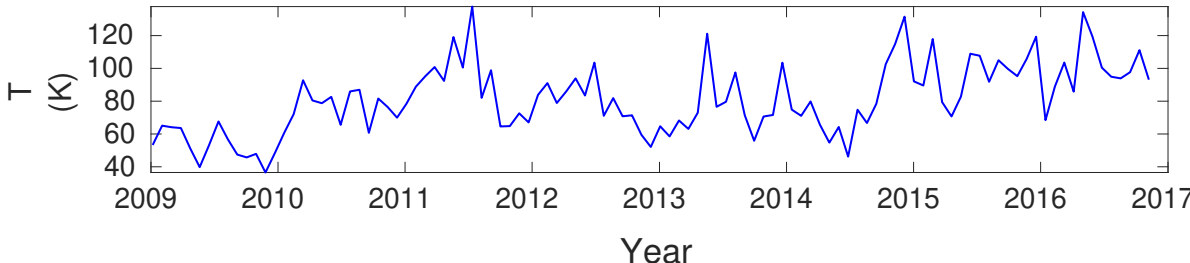

Figure 2. Time series of 27-day-averaged interplanetary magnetic field components and solar wind parameters (IMF and SWP). 
a day. Sources: http://isgi.unistra.fr/indices_kp.php and https://www.ngdc.noaa. gov/stp/geomag/kp_ap.html

Newell et al. (2007), derived a nearly universal coupling function that represents the rate of magnetic flux removed from the dayside magnetopause, $d \Phi_{M P} / d t$ that is itself an electric field. The rate at which magnetic flux is opened at the magnetopause is calculated as a function of the rate at which field lines approach the magnetopause (determined by the solar wind velocity $V$ ). Additionally, they compute the fraction of field lines that merge at magnetosphere (calculated by a sine function of clock angle $\left.\theta_{c}\right)$ and the strength of the transverse IMF $\left(B_{T}\right.$, that is proportional to the amount of flux opened).

In this work we used a version of Newell's function derived by Tsyganenko and Andreeva, 2015, that introduces a normalisation factor of $10^{-4}$ and we denote the merging function as NLL index:

$$
N L L=10^{-4} V^{4 / 3} B_{T}^{2 / 3} \sin ^{8 / 3}\left(\frac{\theta_{c}}{2}\right)
$$

with $V$ in $\mathrm{km} / \mathrm{s}$ and $B_{T}$ in nT, where $B_{T}=\sqrt{B_{Z}^{2}+B_{Y}^{2}}$ is the IMF field component perpendicular to the Sun-Earth axis. Tsyganenko and Andreeva, 2015 computed the NLL index for each 5 min average data record as an average over 30 min long trailing interval, immediately preceding the current time moment. We binned the 5-min data to 27-day resolution in order to compare with the other parameters.

Some of these parameters are displayed in Figure 3.

\section{4. $B_{Z S}$ and $\mathrm{T}$ indices}

Two other types of parameters were tested in this work, as proxies of the interconnection between IMF and geomagnetic activity $\left(B_{Z S}\right)$ and of the imprint of particular magnetospheric current systems into the observed activity on surface (TI).

As is known, (e.g., Schwenn, 2006) the southward IMF $B_{Z}$ is favourable to the energy transport from SW into the Earth's magnetosphere. The daily percentage of the southward component of IMF, hereafter $B_{Z S}$, was calculated from hourly values of IMF $B_{Z}$ in the GSM coordinate system. For day $j$ :

$$
B_{Z S}^{j}=\frac{n^{j} * 100}{24}
$$

where $n^{j}$ is the number of IMF $B_{Z}$ GSM negative (southward) hourly means in day $j$. Then we averaged the daily $B_{Z S}$ over every 27 -days period.

The T-indices were calculated using the $\mathrm{X}$ component of separate contributions from magnetospheric currents on the Earth's surface, from TS05 model simulations. Namely, the X component of the tail current sheet (T-TAIL), the symmetric ring current (T-SRC), the partial ring current (T-PRC) and the field aligned currents (T-FAC). In order to compute T-indices, first all differences between the maximum and minimum (range) values of the corresponding $\mathrm{X}$ 


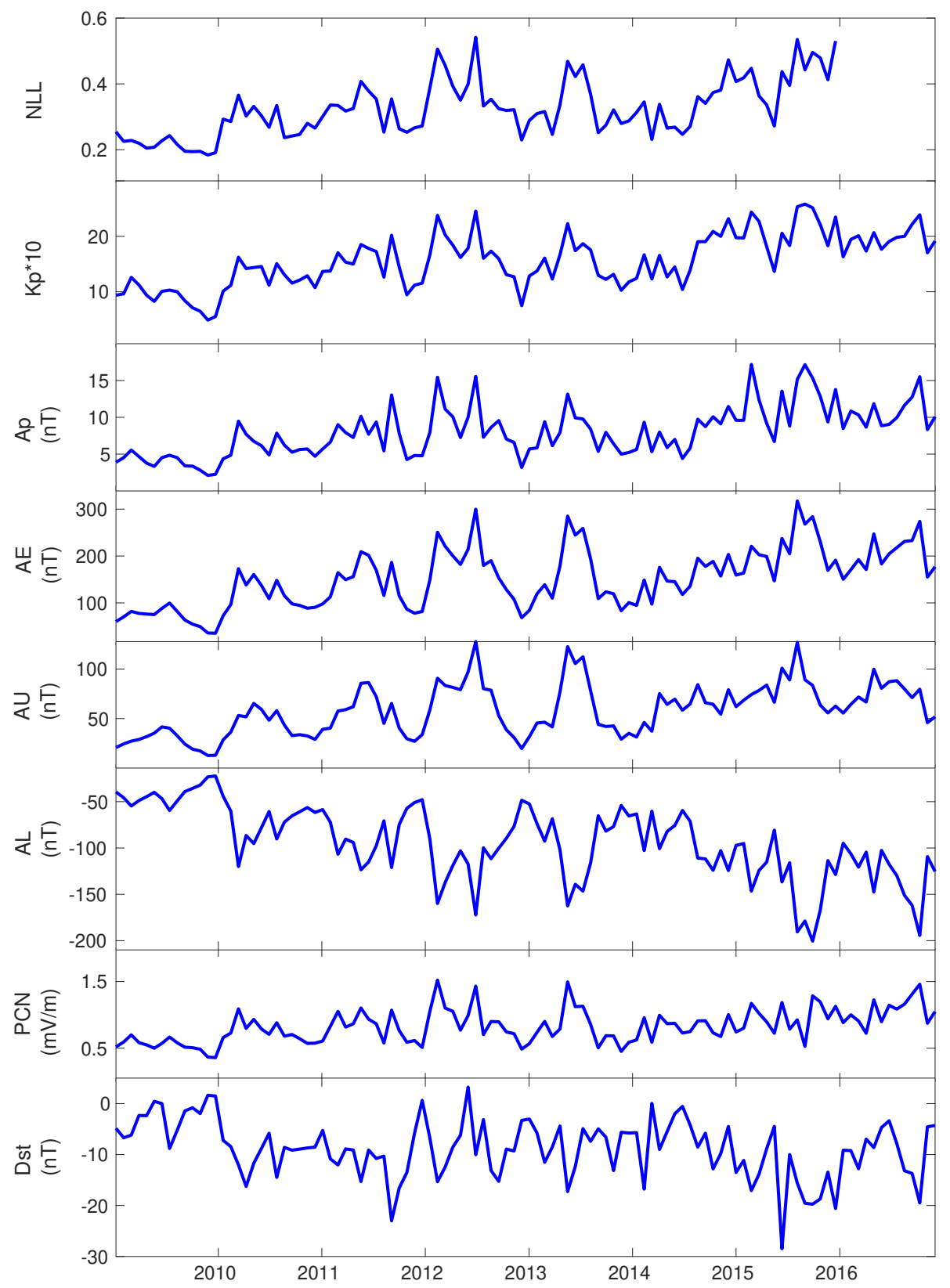

Figure 3. Time series of 27-day-averaged geomagnetic activity indices (GAIs) and coupling function NLL. 
component over each 3-hour time interval were calculated, in nT, a total of 8 values per day:

$$
\Delta T 8_{c u r}^{o b s}(i)=\max \left[T_{c u r}^{i}, T_{c u r}^{i+1}, T_{c u r}^{i+2}\right]-\min \left[T_{c u r}^{i}, T_{c u r}^{i+1}, T_{c u r}^{i+2}\right]
$$

with $i=1,4,7,10,13,16,19$ and $22 . T_{\text {cur }}^{i}$ are hourly values of $T_{\text {cur }}$ for hour $i$. The superscript obs stands for a certain observatory (COI, PAG, NVS and BOU), and the subscript cur stands for a certain current system (TAIL, SRC, PRC or FAC). Then, for each observatory, we computed an averaged daily value as the mean of each group of 3-hour values:

$$
T_{c u r}^{o b s, j}=\frac{1}{8}\left(\Delta T 8_{c u r}^{o b s}(1)+\Delta T 8_{c u r}^{o b s}(4)+\ldots+\Delta T 8_{c u r}^{o b s}(22)\right)
$$

As a third step, we calculated a global daily mean, representative of the behaviour seen at (northern hemisphere) mid-latitudes, using the corresponding daily $T_{c u r}^{o b s, j}$ at all four observatories:

$$
T_{c u r}^{j}=\frac{1}{4}\left(T_{c u r}^{C O I, j}+T_{c u r}^{P A G, j}+T_{c u r}^{N V S, j}+T_{c u r}^{B O U, j}\right)
$$

Finally, we averaged mid-latitude daily values over 27 -day periods. TI series are shown in Figure 4.

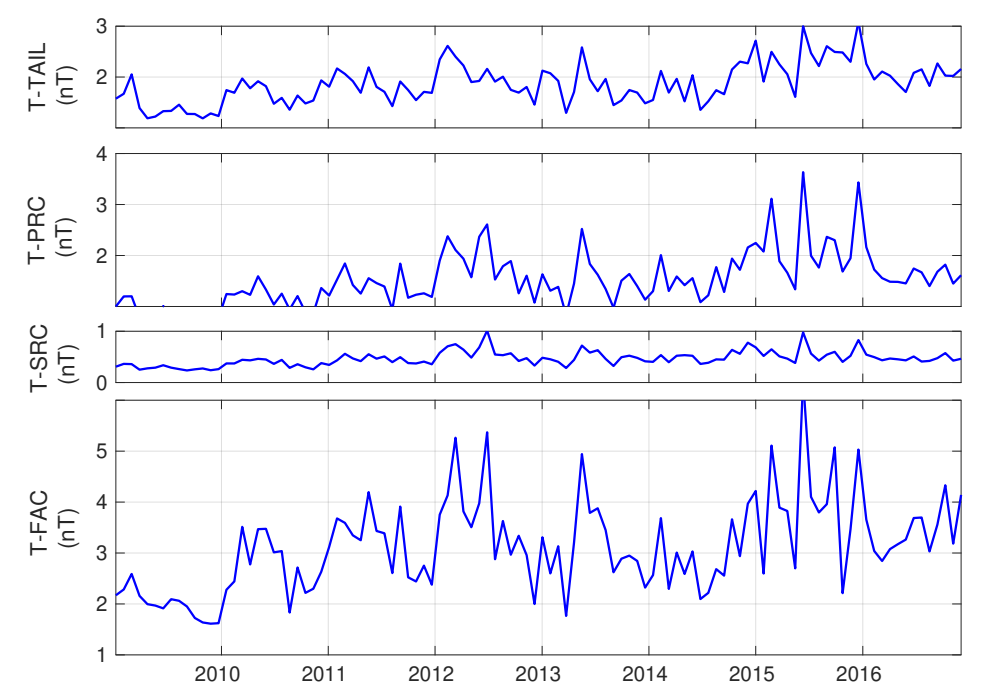

Figure 4. Time series of 27-day-averaged TI magnetospheric indices.

\subsection{Toward-Away Asymmetries}

The orientation of IMF in the plane perpendicular to the Z-axis of the Geocentric Solar Equatorial (GSEq) coordinate system (toward or away from the Sun), is known to also have an impact on geomagnetic activity (e.g., Sabbah, 1995; Zhao 
and Zong, 2012). Considering that the IMF is along the Parker spiral direction, there are two possible polarities, either "away" or "toward" (the Sun). The "away" (or positive) polarity implies IMF $B_{Y}>0$ in the GSEq coordinate system, while the "toward" (or negative) polarity implies IMF $B_{Y}<0$. The IMF polarity close to Earth changes as the Earth crosses the Heliospheric Current Sheet (HCS). To the north of the HCS, the polarity is the same as the solar magnetic polarity which has an oscillating cycle of $22 \mathrm{yrs}$. To address the possible influence of IMF polarity on geomagnetic activity, values of "toward-away" asymmetries were calculated for each of the analyzed parameters (see sections 2.1-2.4).

The Wilcox Solar Observatory (WSO, http://wso.stanford.edu/SB/SB.html) provides a list of well defined sector boundaries around the Sun inside which the IMF has positive or negative polarities, and such that: 1) data is of good quality, 2) the reversal takes place cleanly, and 3) intervals on either side of the boundary have an uniform field direction for $\sim 4$ days. The list is inferred from geomagnetic and spacecraft observations. This WSO boundary list was used to separate days with different polarity signs. When no data were available in the WSO list, "toward" and "away" polarities were calculated using the angle between IMF GSE radial component $\left(\sqrt{B_{X}^{2}+B_{Y}^{2}}\right)$ and the positive X GSE axis (measuring the angle anti-clockwise and using daily means of IMF $B_{X}$ and IMF $B_{Y}$ in the GSE coordinate frame, from NASA/GSFC's OMNI data set through OMNIWeb (see Figure 5) and Sabbah (1995) definition: IMF is "away" from the Sun when the angle is between $45^{\circ}$ and $225^{\circ}$ and is "toward" to the Sun in any other case. Note that the angle between $B_{Y}$ GSE and $B_{Y}$ GSEq in the two coordinate systems is small, of $\sim 7^{\circ}$, so they are sometimes used interchangeably.

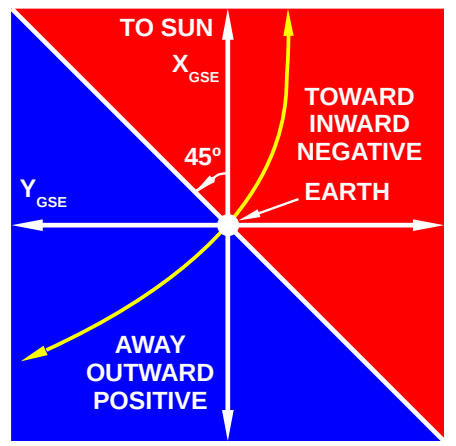

Figure 5. Definition of away and toward polarity seen from the Earth. Angles in $\left[45^{\circ}, 225^{\circ}\right]$ are considered away (outward) or positive polarity. Other angles are considered toward (inward) or negative polarity (Sabbah, 1995). Yellow arrows represent the IMF's trajectory and direction in the azimuthal GSE framework. 
Figure 6 shows our pixel plot of 'toward' and 'away' days from 2009 to 2016, obtained using Wilcox Solar Observatory list of sector boundaries combined with calculation of toward and away days using Sabbah (1995) criteria. From January 2009 to March 2012 the solar magnetic field has negative polarity (44 Bartels' rotations); then, from April 2012 to March 2015 there is a transition epoch (40 rotations), and, finally, from April 2015 to 2016 the solar magnetic field has positive polarity (24 rotations). The Sun's North (South) pole is most inclined toward the Earth in fall (spring), therefore, the dominant polarity seen by the Earth around fall is that of the Sun (and the opposite around spring).

The "toward-away" asymmetries (with respect to IMF polarity) of the 33 parameters were computed as follows. For each parameter mentioned in sections 2.1-2.4, we split values into two groups, and calculate separate averages for days when the IMF is directed toward the Sun (T), and days when the IMF is directed away from the Sun (W), and computed the two values:

$$
\bar{T}=\frac{1}{n} \sum_{i=1}^{n} T^{i} \quad \bar{W}=\frac{1}{m} \sum_{i=1}^{m} W^{i}
$$

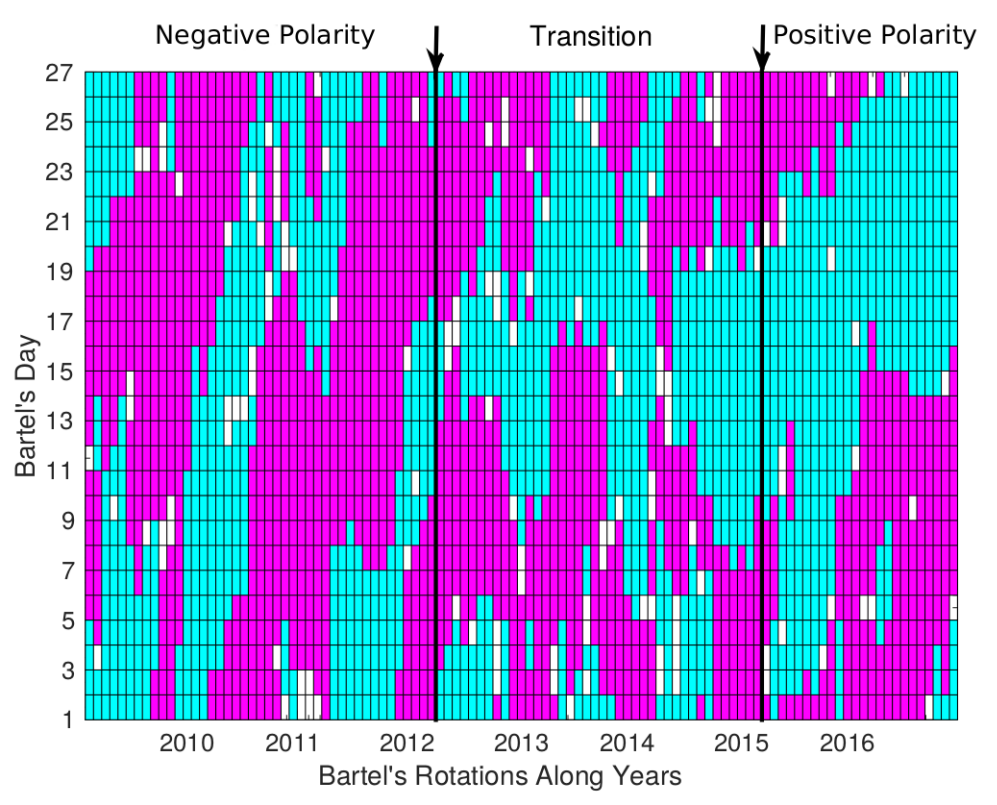

Figure 6. Pixel plot of days with toward/negative polarity (pink) and away/positive polarity (cyan) IMF. White days means missing data or polarity that could not be determined. Each column is a solar rotation (27 days) in the period 2009-2016. The solar magnetic field has negative polarity in 2009 - 2012 and has positive polarity in 2015 - 2016. The interval $2012-$ 2015 is a transition period.

where $\bar{T}$ and $\bar{W}$ are mean values of a certain parameter over a Bartel's rotation period, counting only "toward" days in the former case (in a total of $n$ ) and only "away" days in the later (in a total of $\mathrm{m}$ ). Then, for each parameter, we 
obtained the asymmetry A over each Bartel's period as the difference between the corresponding "toward" and "away" means:

$$
A=\bar{T}-\bar{W}
$$

The standard error for each $A$ was calculated as:

$$
\sigma_{A}=\sqrt{\frac{\sigma_{T}^{2}}{n}+\frac{\sigma_{W}^{2}}{m}}
$$

where $\sigma_{T}$ is the standard deviation of parameter values during "toward" days, and $\sigma_{W}$ is the standard deviation of parameter values during "away" days. Asymmetries were standardised $\left(A^{s}=A / \sigma_{A}\right)$.

Figure 7 shows, as an example, a plot of $A$ asymmetries for $B_{Z}$ GSM, the Newell coupling function, the T-FAC index and different GAI parameters. Note that $A$ for $B_{Z}$ GSM and Dst were multiplied by -1 to facilitate the comparison. The percentage of toward-to-the-Sun days in each Bartel's rotation is also shown, at the top-left of the figure. 

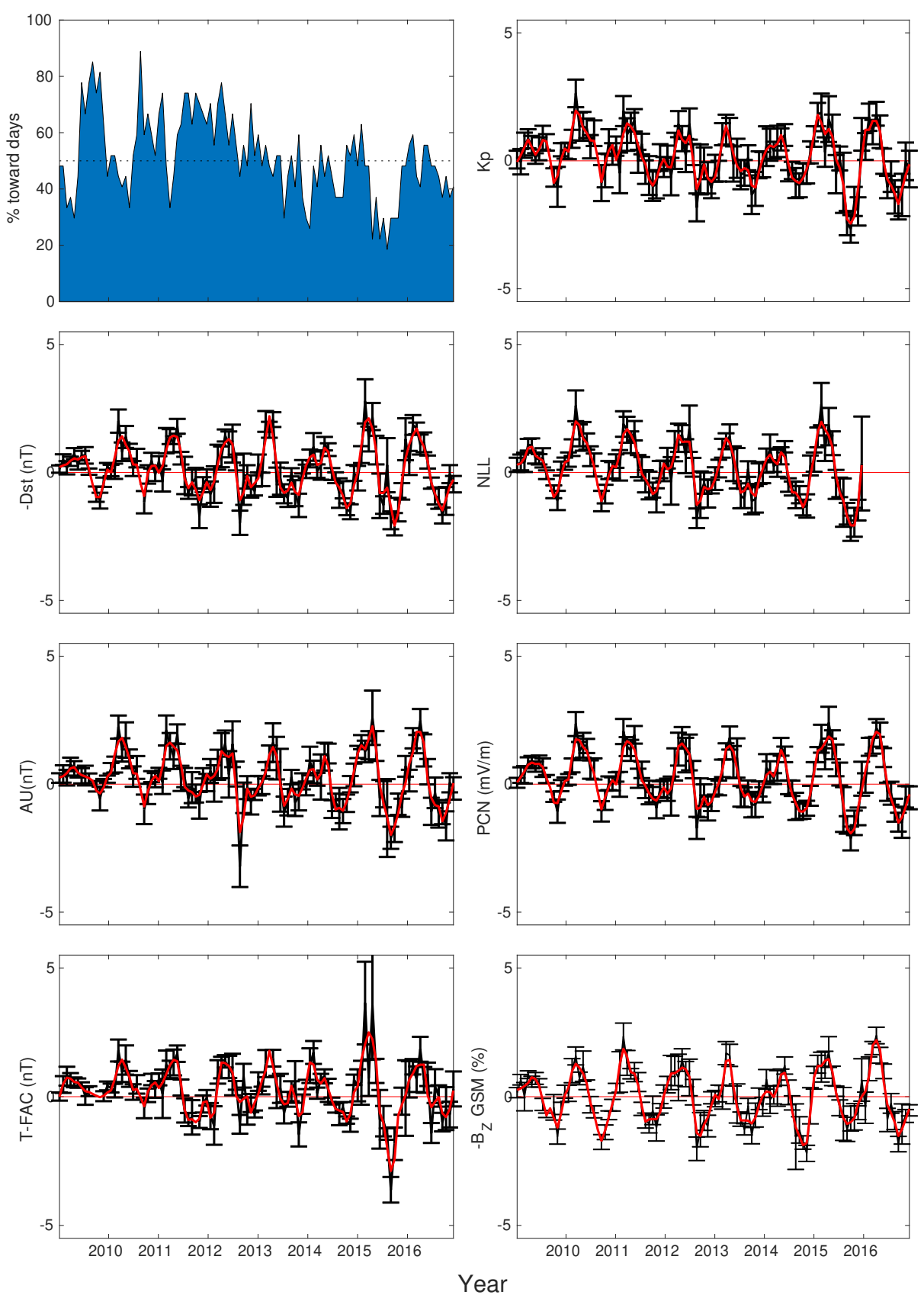

Figure 7. Top-left: Percentage of days with toward IMF during each Bartel's rotation for the whole 2009-2016 period. Top-right: Time series of the asymmetry $A$ for the Kp index. From top to bottom, left to right: time series of $A$ for the - Dst, the coupling function NLL, AU, PCN, T-FAC and $-B_{Z}$ GSM, with corresponding error bars. The smooth red curves represent a LOESS regression model, using span $=0.5$. 


\section{Results and Discussion}

In this section we present a correlation analysis of all the solar, solar wind and geomagnetic field parameters and their asymmetries. Spearman's cross-correlation coefficients were calculated for the 27-day means for the 2009-2016 time interval, except in the case of the Newell's coupling function, available only until 2015.

\subsection{Correlation Analysis of 27-Day-Averaged Series}

Spearman's cross correlations $\mathbf{r}_{\mathbf{S}}$ between the 33 parameters, averaged over 27 day intervals, were computed in order to search for a pattern that explains the physical processes involved in the solar wind-magnetosphere coupling. Table 3 shows the correlations obtained. As a criteria for choosing the 'best' correlations, only values $\left|r_{S}\right| \geq 0.4$ and p-value $p \leq 0.05$ are shown.

FA-N and FA-S are the solar parameters with highest (in absolute value) correlation coefficients with parameters of other groups, and represent the activity in the Sun's chromosphere. They give us a valuable picture of the solar dynamo, vital in understanding the global structure of the Heliospheric Magnetic Field. At solar minimum (near 2009), the Sun has few facular areas (but never zero facular area, as can be seen from Figure 1), corresponding with a mostly dipolar magnetic field, with a slow wind at the solar equator and a fast wind at the poles. At maximum of activity (around 2014), the Sun has larger facular areas, with a multipolar magnetic field and a bi-modal wind distribution at all latitudes (Perri et al., 2018). FA-S is the parameter with more significant correlation coefficients among all solar parameters.

Among the solar wind parameters, the 27-day mean of the total field B is the parameter that has more significant correlations and the highest sum of correlations with other parameters.

The $B_{Z S}$ GSM, a 27-day average of the percentage of southward $B_{Z}$ GSM occurrences, correlates with GAI and TI parameters better than the 27-day averaged $B_{Z}$ GSM. In fact, at this time resolution, correlations of $B_{Z}$ GSM with most of GAI and TI parameters are less than 0.4. This shows that, in spite of the recurrent use of $B_{Z}$ GSM as a proxy for geomagnetic storms (with a timescale much shorter than the one resolved here), $B_{Z S}$ GSM is a better proxy of magnetospheric activity than $B_{Z}$ GSM, at the 27-day time resolution.

NLL is the combination of SW speed and IMF components which, among similar combinations, more closely represents the solar wind-magnetospheric coupling (Newell et al., 2007). It shows significant correlations with all the other parameters, in Table 3.

Among GAI parameters, Kp presents the best scores. Finally, regarding Tindices, T-SRC and T-PRC have highest (in absolute value) correlation coefficients with parameters from other groups. They represent the variations in the symmetric and the partial ring currents, respectively, due to energetic particles injected by solar wind disturbances, i.e., ICMEs. T-PRC and T-SRC associate the ring current activity to chromospheric and photospheric activity, better than GAI parameters. Note in particular that Dst, a standard ring current index, has no significant correlations with solar parameters. Also, Dst shows lower 
correlations with B and the coupling function NLL than the indices T-SRC and T-PRC.

Comparing the trends of these parameters is of particular interest for identification of causal relashionships between solar and terrestrial phenomena.

\subsection{Analysis of 27-Day-Mean Series of Asymmetries}

One interesting result of the analysis of the asymmetry parameters variations is the annual variation in 27-day asymmetries that sorts out in GAI parameters and NLL coupling function (see Figure 7), but also in $B_{Z S}$ GSM and $B_{Z}$ GSM. Asymmetries for different parameters were computed as explained in Section 2.5. For NLL, AU, PCN, T-FAC and Kp we see positive asymmetries at the first half of the year and negative at the second half, with maxima/minima near the equinoxes and zero asymmetry near the solstices. The same for $-B_{Z}$ GSM, - Dst, since these values have the opposite sign. It means that every year the values of these parameters are larger when IMF points toward (away) the Sun than the values when IMF points away (toward) the Sun in the first (second) half of the year. It agrees with Zhao and Zong (2012), who found that geomagnetic activity is much more intense around spring equinox when the direction of IMF is toward the Sun, while much more intense around fall equinox when the direction of IMF is away the Sun.

These observations are in agreement with the Russell-McPherron (R-M) effect (Russell and McPherron, 1973) proposed to explain the semiannual variation of geomagnetic activity (SAV). The IMF tends to be parallel to the solar wind blown from the Sun, and as a result it lies mostly over the solar equatorial plane (X-Y plane in the GSEq frame) (e.g. Koskinen, 2011). On the other hand, the probability of reconnection between the IMF and the Earth's field increases for larger values of the projection of IMF along - Z GSM axis. In this context, Russell and McPherron (1973) suggested that the relevant parameter to measure the probability of geomagnetic activity could be the $\theta_{R M}$ angle between Z-GSM and Z-GSEq axes: the larger $\theta_{R M}$, the more the solar equatorial plane is tilted relative to the GSM magnetospheric equatorial plane, increasing the probability for reconnection. Both coordinate systems share the same X-axis (line pointing from the Earth to the Sun), and as a result $\theta_{R M}$ is measured in the Y-Z plane of both GSM and GSEq reference frames (see Figure 8).

The R-M mechanism could explain the observed SAV, since $\theta_{R M}$ attains larger (absolute) values close to the equinoxes (more exactly, begin of April and October) (e.g. Lockwood et al., 2020). There are, nonetheless, two other mechanisms proposed to explain SAV (e.g. Poblet, Azpilicueta, and Lam, 2020; Lockwood et al., 2020): the axial hypothesis, that states the main cause is the position of the Earth with respect to the solar equator (the heliographic latitude), with maximum near the equinoxes $\left( \pm 7.5^{\circ}\right)$ and minimum near solstices $\left(\sim 0^{\circ}\right)$; the equinoctial hypothesis, that identifies as the most relevant parameter the angle between the Earth-Sun line and the geomagnetic dipole axis of the Earth (the tilt angle, or its complement).

Zhao and Zong (2012) noticed that, if R-M is the dominant mechanism behind $\mathrm{SAV}$, a toward/away asymmetry should be seen in geomagnetic activity. For 


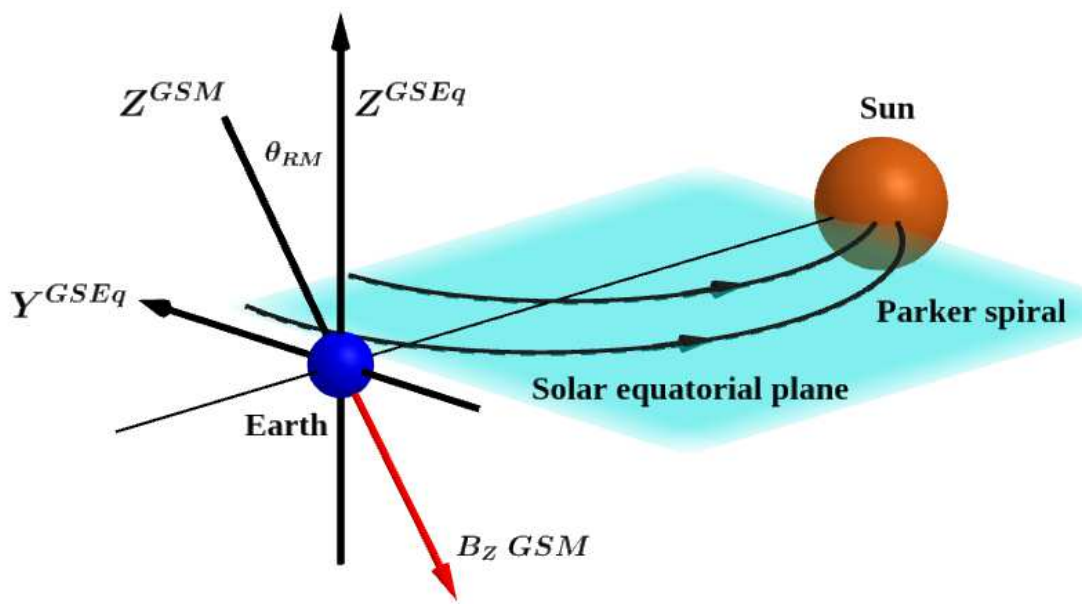

Figure 8. The $\theta_{R M}$ during the spring equinox, with IMF toward the Sun. The $B_{Z}$ GSM southward (red vector) increases due to the contribution of the negative $B_{Y}$ GSEq. Figure adapted from Poblet, Azpilicueta, and Lam (2020)

the same $\theta_{R M}$ angle, the IMF $B_{Z}$-GSM projection can be positive or negative depending if the IMF polarity is toward or away. As shown by them, during fall equinox when $\theta_{R M}$ attain lowest negative values $\left(\sim-26^{\circ}\right)$, the IMF $B_{Z}$-GSM is negative for away IMF polarity and positive otherwise. During spring equinox, when $\theta_{R M}$ attains highest positive values $\left(\sim 26^{\circ}\right)$, the IMF $B_{Z}$-GSM is negative for toward IMF polarity and positive otherwise. In this way, the toward/away asymmetry dependence of SAV introduces an asymmetry between spring and fall equinoxes and an annual periodicity should be seen in SAV.

As it is seen in the top-left of Fig. 7 the days with toward IMF prevail in 2009-2012 and days with away IMF prevail in 2015-2016, coinciding with the polarity of Sun's northern hemisphere in both time intervals. This means that the Earth was located north of the heliospheric current sheet most of the time. It is the Earth's prevailing position since solar cycle 16 (Mursula and Hiltula, 2003).

We identify the annual oscillation in 27-day-mean values of the asymmetry for GAI parameters and for NLL coupling function, as shown in Figure 7 . The $B_{Z^{-}}$ GSM and $B_{Z S}$-GSM parameters also show a similar oscillation. Figure 7 further shows that during the 2009-2012 period of solar negative/toward polarity the asymmetry has higher positive/away values in spring (and less negative values in fall), and in the 2015-2016 period when the solar polarity is positive, the asymmetry has also higher positive values in spring (and more negative values in fall). This behaviour can in principle be explained due to the R-M mechanism, although a larger dataset, including several solar polarity inversions, would be needed to draw a more robust conclusion.

In search for the ubiquitous presence (or not) of the annual oscillation in series of toward/away asymmetries, Table 4 shows the correlation between the Kp asymmetry, the $B_{Z}$ GSM, the $B_{Z S}$ GSM, three solar wind parameters, 
the NLL, the GAI and the TI-indices asymmetries. When computing crosscorrelations with that curve we are evaluating the presence of a synchronized annual oscillation in all those different parameters, because the $A \mathrm{Kp}$ fits better with a sine wave. The correlation would also be low with other IMF parameters and solar parameters. 
Table 3. Spearman's cross-correlation coefficients of 33 Bartel's means, with $\left|r_{s}\right| \geq 0.4$ and $p \leq 0.05$

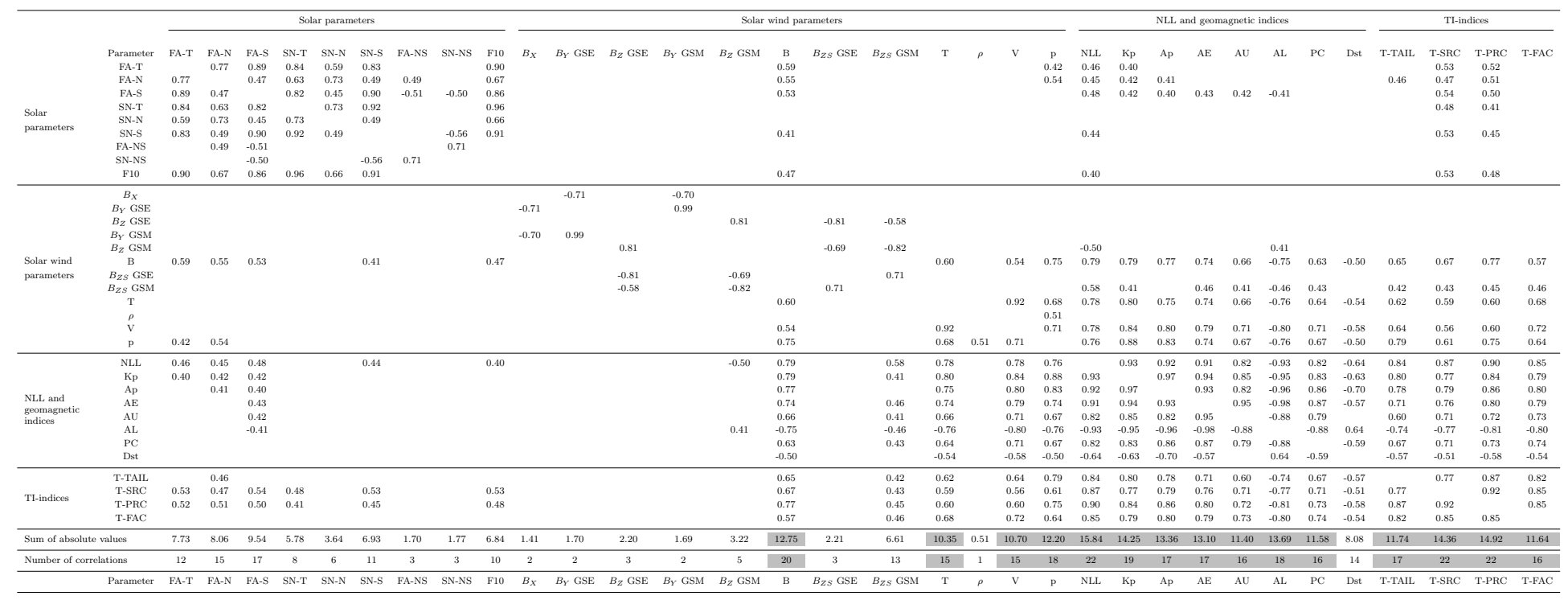


Table 4. Spearman's correlation coefficients between Kp asymmetries and asymmetries of other parameters.

\begin{tabular}{ll}
\hline Parameter & Correlation coefficient \\
\hline $\mathrm{Ap}$ & 0.97 \\
$\mathrm{AE}$ & 0.93 \\
$\mathrm{AL}$ & -0.92 \\
$\mathrm{AU}$ & 0.91 \\
$\mathrm{NLL}$ & 0.91 \\
PC & 0.89 \\
Dst & -0.82 \\
T-FAC & 0.75 \\
$B_{Z S}$ GSM & 0.72 \\
$B_{Z}$ GSM & -0.70 \\
T-SRC & 0.69 \\
SPEED & 0.66 \\
T-PRC & 0.62 \\
TEM & 0.60 \\
T-TAIL & 0.59 \\
PRES & 0.50 \\
\hline
\end{tabular}

\section{Conclusion}

In order for the global dynamical state of the Sun to influence the Earth, some time should elapse. This study considers time averages over whole Bartel's rotation periods (27 Earth days), for different parameters involved in Sun-Earth interaction processes.

We computed hourly synthetic series of the $X$ component of four magnetospheric current systems at Earth's surface, in the 2009-2016 time period, using the TS05 model at the geomagnetic coordinates of four magnetic observatories in mid-latitudes $\left(\sim 40^{\circ} \mathrm{N}\right)$. Four new mid-latitude geomagnetic indices (T-indices) were derived from these series: T-TAIL (from tail current), T-SRC (symmetric ring current), T-PRC (partial ring current) and T-FAC (field aligned current). Each T-index measures the contribution to the geomagnetic activity of its corresponding current field. The T-SRC and T-PRC indices have better correlations with solar parameters than other geomagnetic activity indices and solar wind parameters. A relation between solar parameters and the ring current system is inferred from these results. We also calculated the $B_{Z S}$ GSM, as the percentage 
of hourly occurrences of the southward $B_{Z}$ GSM in a day. It is a possible substitute for the most often used $B_{Z}$ GSM, as an estimator of the magnetospheric activity at the 27-day time resolution.

The geomagnetic activity observed during solar cycle 24 was largely driven by high speed solar wind streams (e.g., Gerontidou, Mavromichalaki, and Daglis, 2018; Richardson, Cliver, and Cane, 2002). As a result, strong correlations were expected between solar wind temperature, speed and ram pressure, and geomagnetic activity indices as those tested here. Although those solar wind parameters correlate strongly with GAI (see Table 3), they do not show as high correlations with solar surface parameters as does the IMF B intensity.

We analysed the behaviour of the working parameters with respect to the toward/away asymmetry, in search for some possible influence of the solar magnetic field polarity over geomagnetic activity. We identified an annual oscillation in 27day-mean values of geomagnetic activity indices and also Newell coupling function, as shown in Figure 7 . The $-B_{Z}$-GSM and $B_{Z S}$-GSM parameters also show the same oscillation. We think this result favours the Russell-McPherron mechanism as the dominant mechanism explaining semiannual variation of geomagnetic activity.

The main conclusions derived from the analysis presented above, based on the correlation analysis of a large data set of parameters relating the different stages of Sun-Earth interaction processes, are the following:

- Hemispheric facular areas computed as averages over 27-day periods show better correlations with solar wind parameters (in particular the total IMF field B) and with geomagnetic activity indices (in particular Kp) than other solar parameters as the hemispheric sunspot numbers or the solar radio flux F10.7 cm. Furthermore, they evolve in a more continuous way along the solar cycle than sunspots, never getting to zero even at solar minimum.

- T-SRC and T-PRC indices are new parameters defined here to represent the dynamics of the ring current. They correlate better with solar parameters than the Dst index, which is more frequently used as a proxy for the energy of the ring current. The Newell's coupling function computed from solar wind and IMF parameters, and the planetary index of global geomagnetic activity $\mathrm{Kp}$, both show significant correlations with a large number of other proxies. Nonetheless, their correlation with solar surface parameters is lower than what is obtained using the newly defined magnetospheric activity proxies T-SRC and T-PRC.

- The $B_{Z S}$ GSM index defined in this study, seems to be a better choice than $B_{Z}$ GSM to characterise magnetospheric activity at the 27-day time resolution, since it shows higher correlations with all GAI parameters.

- The total field $B$ averaged over 27-days is the solar wind parameter that correlates the best with solar surface parameters, in particular facular areas.

- The annual oscillation found in series of toward/away asymmetry for the GAI parameters, NLL and both $B_{Z}$ GSM and $B_{Z S}$ GSM parameters seems 
to support that the Russell-McPherron mechanism is the main mechanism explaining SAV.

- Further support of the R-M mechanism is the variation of amplitude of the toward/away asymmetry with the solar magnetic field polarity, which is also retrieved in our results. Our results suggest that, for the analysed 2009-2016 period that covers the two different solar polarities, solar positive polarity leads to geomagnetic activity more intense during fall equinox and negative polarity leads to geomagnetic activity more intense during spring equinox.

Finally, the new indices proposed here (T-indices and $B_{Z S}$ GSM) should be tested further in other solar cycles to strength their use as proxies of the SunEarth interactions. It would be particularly interesting to follow the changes and inter-correlations of all these parameters (in particular $B, B_{Z S}$ GSM, FA-N, FA$\mathrm{S}$, TI and NLL) in the coming years of the new cycle and investigate the main physical mechanisms involved.

Acknowledgments We are grateful to N. Tsyganenko for kindly answering our questions about his model and for making his codes easily available to the science community. We acknowledge use of NASA/GSFC's Space Physics Data Facility's OMNIWeb service, and OMNI data. We acknowledge to Teresa Barata and her collaborators for their courtesy to share with us the plages data computed by them. CITEUC is funded by National Funds through FCT - Foundation for Science and Technology (Projects UID/00611/2020 and UIDP/00611/2020) and FEDER - European Regional Development Fund through COMPETE 2020 Operational Program Competitiveness and Internationalization (project: POCI-01-0145-FEDER-006922). J. Fernandes acknowledges funding from POCH and Portuguese FCT grant SFRH/BSAB/143060/2018 and from the project ReNATURE - Valuation of Endogenous Natural Resources in the Central Region (CENTRO-01-0145-FEDER-000007). Y.C. was supported by the Erasmus Mundus Action 2 Consortium AMIDILA, Lot 15 - strand 1, 2013-2588/001-001-EM Action 2 Partnerships. A.L.M. is supported by FCT - Foundation for Science and Technology, post-doc grant SFRH/BPD/74812/2010. F. J. G. Pinheiro was funded through project UID/Multi/00611/2013. This study could also benefit from project MAG-GIC: PTDC/CTA-GEO/31744/2017 funded by FCT.

\section{References}

Ballester, J.L., Oliver, R., Carbonell, M.: 2005, The periodic behaviour of the NorthSouth asymmetry of sunspot areas revisited. Astron. Astrophys. 431, L5. DOI. ADS. [2005A\&A . .431L ..5B]

Barata, T., Carvalho, S., Dorotovic, I., Pinheiro, F.J.G., Garcia, A., Fernandes, J., Lourenco, A.M.: 2018, Software tool for automatic detection of solar plages in the Coimbra Observatory spectroheliograms. Astronomy and Computing 24, 70. [2018A\&C....24...70B]

Beggan, C.D., Beamish, D., Richards, A., Kelly, G.S., Thomson, A.W.P.: 2013, Prediction of extreme geomagnetically induced currents in the UK high-voltage network. Space Weather J. 11, 407. [Beggan13]

Buonsanto, M.J.: 1999, Ionospheric Storms - A Review. Space Sc. Rev. 88, 563. [Buon99] 
Castillo, Y.S., Pais, M.A., Fernades, J., Ribeiro, P., Morozova, A.L., Pinheiro, F.J.G.: 2017, Geomagnetic activity at Northern Hemisphere's mid-latitude ground stations: How much can be explained using TS05 model. Journal of Atmospheric and Solar-Terrestrial Physics 165-166, 38. [Castillo17]

Davis, T.N., Sugiura, M.: 1966, Auroral electrojet activity index AE and its universal time variations. Journal of Geophysical Research (1896-1977) 71(3), 785. DOI. https://agupubs. onlinelibrary.wiley.com/doi/abs/10.1029/JZ071i003p00785. [Sugiura66]

Dorotovic, I., Rybak, J., Garcia, A., Journoud, P.: 2010, North-south asymmetry of Ca II K regions determined from OAUC spectroheliograms: 1996 - 2006, 58. [Dorotovic10]

Georgieva, K., Kirov, B., Tonev, P., Guineva, V., Atanasov, D.: 2007, Long-term variations in the correlation between NAO and solar activity: The importance of north south solar activity asymmetry for atmospheric circulation. Advances in Space Research 40, 1152. DOI. ADS. [2007AdSpR . 40.1152G]

Gerontidou, M., Mavromichalaki, H., Daglis, T.: 2018, High-Speed Solar Wind Streams and Geomagnetic Storms During Solar Cycle 24. Solar Physics 293(131). [Gerontidou2018]

Gonçalves, E., Mendes-Lopes, N., Dorotovič, I., Fernandes, J.M., Garcia, A.: 2014, North and South Hemispheric Solar Activity for Cycles 21 - 23: Asymmetry and Conditional Volatility of Plage Region Areas. Solar Phys. 289, 2283. DOI. ADS. [2014SoPh. .289.2283G]

Janardhan, P., Fujiki, K., Ingale, M., Bisoi, S.K., Rout, D.: 2018, Solar cycle 24: an unusual polar field reversal. ArXiv e-prints. ADS. [2018arXiv180506654J]

Janardhan, P., Fujiki, K., Ingale, M., Bisoi, S.K., Rout, D.: 2018, Solar cycle 24: An unusual polar field reversal. Astronomy \& Astrophysics 618(A148). [Janardhan2018]

Joshi, B., Bhattacharyya, R., Pandey, K.K., Kushwaha, U., Moon, Y.-J.: 2015, Evolutionary aspects and north-south asymmetry of soft X-ray flare index during solar cycles 21,22 , and 23. Astron. Astrophys. 582, A4. DOI. ADS. [2015A\&A...582A . . 4J]

King, J.H., Papitashvili, N.E.: 2005, Solar wind spatial scales in and comparisons of hourly Wind and ACE plasma and magnetic field data. Journal of Geophysical Research 110(A02104). [King2005]

Koskinen, H.: 2011, Physics of Space Storms: From the Solar Surface to the Earth, Springer, Germany. ISBN 987-3-642-00310-3. DOI. [Koskinen2011]

Laundal, K.M., Richmond, A.D.: 2016, Magnetic Coordinate Systems. Space Sc. Rev.. [Laundal16]

Lekshmi, B., Nandy, D., Antia, H.M.: 2018, Asymmetry in Solar Torsional Oscillation and the Sunspot Cycle. Astrophys. J. 861, 121. DOI. ADS. [2018ApJ . .861..121L]

Lockwood, M., Owens, M.J., Barnard, L.A., Haines, C., Scott, C.J., McWilliams, K.A., Coxon, J.C.: 2020, Semi-annual, annual and Universal Time variations in the magnetosphere and in geomagnetic activity: 1. Geomagnetic data. Journal of Space Weather and Space Climate 10, 23. DOI. ADS. [Lockwood2020]

Mayaud, P.N.: 1980, Derivation, Meaning, and Use of Geomagnetic Indices. Washington DC American Geophysical Union Geophysical Monograph Series 22. [Lockwood2020]

Menvielle, M., Iyemori, T., Marchaudon, A., Nose, M.: 2011, Geomagnetic Indices. In: Mandea, M., Korte, M. (eds.) Geomagnetic Observations and Models, IAGA Special Sopron Book Series 5, Springer, Dordrecht, 183. [Menv11]

Mordvinov, A.V., Pevtsov, A.A., Bertello, L., Petrie, G.J.D.: 2016, The reversal of the Sun's magnetic field in cycle 24. Solar-Terrestrial Physics 2(1), 3. [Mordvinov16]

Mursula, K., Hiltula, T.: 2003, Bashful ballerina: Southward shifted heliospheric current sheet. Geophysical Research Letters 30(22). [Mursula2003]

Nepomnyashchikh, A., Mandal, S., Banerjee, D., Kitchatinov, L.: 2019, Can the long-term hemispheric asymmetry of solar activity result from fluctuations in dynamo parameters? Astronomy \& Astrophysics 625(A37). [Nepom2019]

Newell, P.T., Sotirelis, T., Liou, K., Meng, C.-I., Rich, F.J.: 2007, A nearly universal solar windmagnetosphere coupling function inferred from 10 magnetospheric state variables. Journal of Geophysical Research: Space Physics 112(A1), n/a. A01206. [Newel107]

Norton, A.A., Gallagher, J.C.: 2009, Solar-Cycle Characteristics Examined in Separate Hemispheres: Phase, Gnevyshev Gap, and Length of Minimum. Solar Physics 261(1), 193. [Norton09]

Perri, B., Brun, A.S., Réville, V., Strugarek, A.: 2018, Simulations of solar wind variations during an 11-year cycle and the influence of north-south asymmetry. Journal of Plasma Physics 84(5), 765840501. DOI. ADS. [Perri18]

Poblet, F.L., Azpilicueta, F., Lam, H.-L.: 2020, Semiannual variation of Pc5 ultra-low frequency (ULF) waves and relativistic electrons over two solar cycles of observations: comparison 
with predictions of the classical hypotheses. Annales Geophysicae 38(5), 953. DOI. ADS. [Poblet2020]

Richardson, I.G., Cliver, E.W., Cane, H.V.: 2002, Interplanetary coronal mass ejections in the near-Earth solar wind during 1996-2002Long-term trends in interplanetary magnetic field strength and solar wind structure during the twentieth century. Journal of Geophysical Research 107(A10). [Richardson2002]

Russell, C.T., McPherron, R.L.: 1973, Semiannual variation of geomagnetic activity. Journal of Geophysical Research (1896-1977) 78(1), 92. DOI. https://agupubs.onlinelibrary.wiley.com/ doi/abs/10.1029/JA078i001p00092. [RusselMcPherron73]

Sabbah, I.: 1995, North-South asymmetry of the daily interplanetary magnetic field spiral during the period: 1965 1990. Earth Moon and Planets 70, 173. [Sabbah95]

Schrijver, C.J.: 2015, Understanding space weather to shield society: A global road map for 2015-2025 commissioned by COSPAR and ILWS. Adv. in Space Res. 55, 2745. [Schr15]

Schüssler, M., Cameron, R.H.: 2018, Origin of the hemispheric asymmetry of solar activity. ArXiv e-prints. ADS. [2018arXiv180710061S]

Schwenn, R.: 2006, Space Weather: The Solar Perspective. Living Rev. Solar Phys. 3(2). [Schwenn06]

Sugiura, M.: 1964, Hourly values of the equatorial Dst for IGY. Ann. Int. Geophys. Year 35, 945. [Schwenn06]

Temmer, M., Veronig, A., Hanslmeier, A.: 2002, Hemispheric Sunspot Numbers Rn and Rs : Catalogue and N-S asymmetry analysis. Astronomy \& Astrophysics 390, 707. [Temmer02]

Temmer, M., Rybák, J., Bendík, P., Veronig, A., Vogler, F., Otruba, W., Pötzi, W., Hanslmeier, A.: 2006, Hemispheric sunspot numbers Rn and Rs from 1945-2004: catalogue and N$\mathrm{S}$ asymmetry analysis for solar cycles 18-23. Astron. Astrophys. 447, 735. DOI. ADS. [2006A\&A . . 447 . .735T]

Thomson, A.W.P.: 2012, Space weather applications of Geomagnetic Observatory Data. British Geological Survey. [Thomson12]

Tsyganenko, N.A., Andreeva, V.A.: 2015, A forecasting model of the magnetosphere driven by an optimal solar wind coupling function. J. Geophys. Res. 120, 8401. [TA15]

Tsyganenko, N.A., Sitnov, M.I.: 2005, Modeling the dynamics of the inner magnetosphere during strong geomagnetic storms. J. Geophys. Res. 110, A03208. [TS05]

Waldmeier, M.: 1971, The asymmetry of solar activity in the years 1959 - 1969. Solar Physics 20(2), 332. [Waldmeier71]

Zhao, H., Zong, Q.G.: 2012, Seasonal and diurnal variation of geomagnetic activity: RussellMcPherron effect during different IMF polarity and/or extreme solar wind conditions. Journal of Geophysical Research 117. [Zhao12] 
Figures

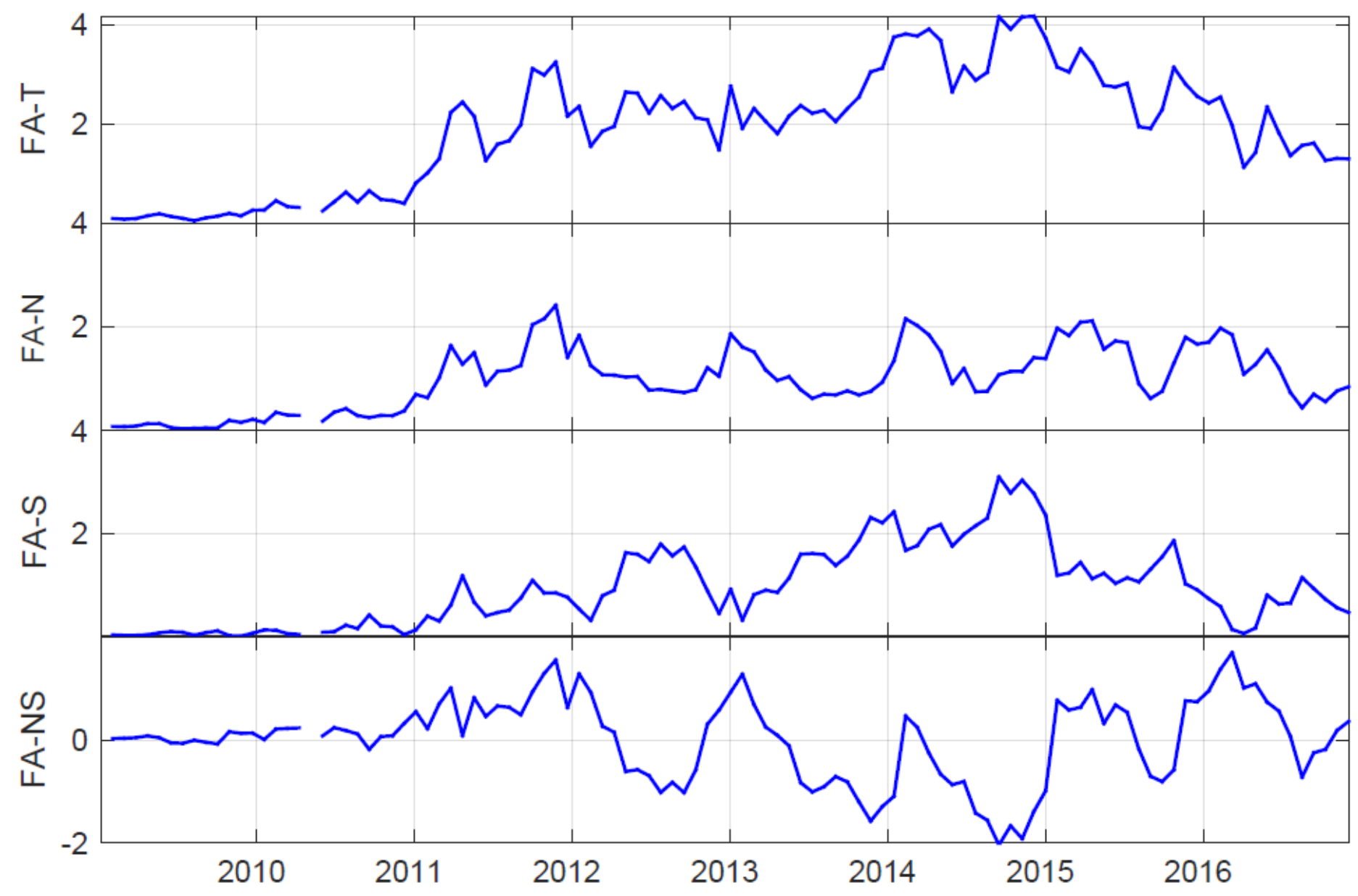

Figure 1

Time series of 27-day-averaged facular areas, in percentage of total solar disk area. 


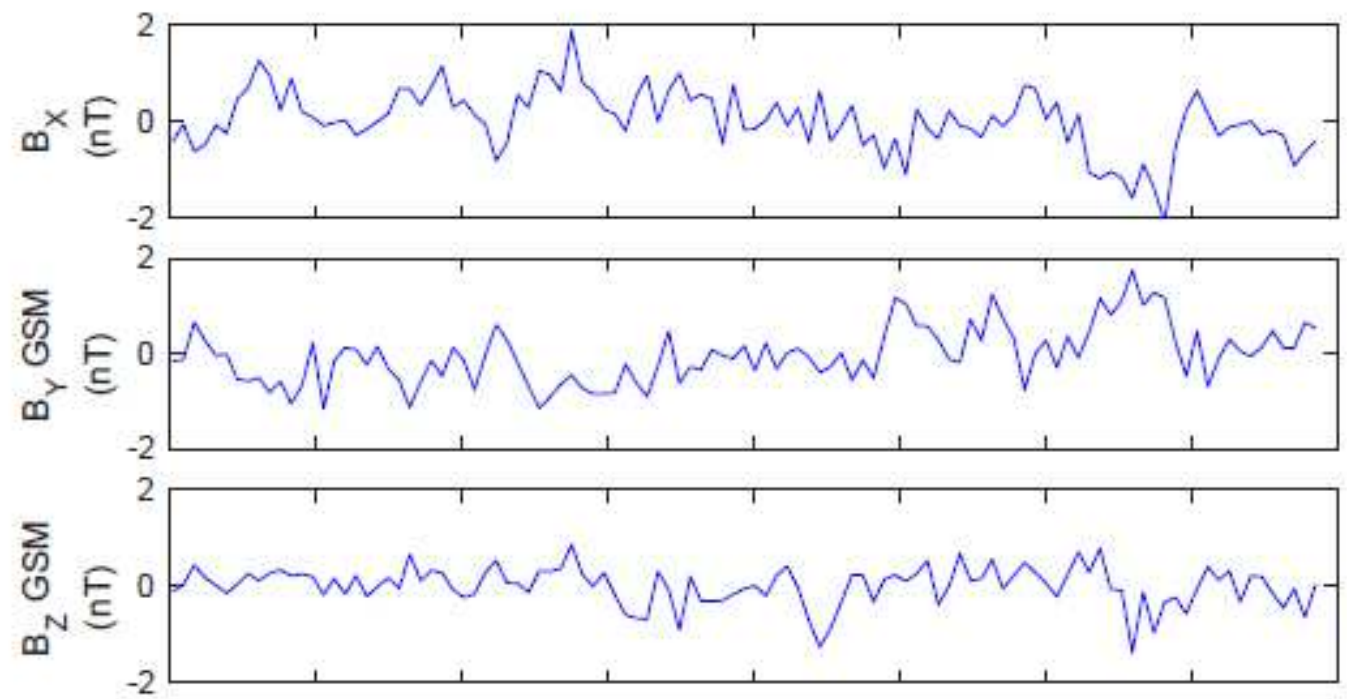

$m E^{4}$

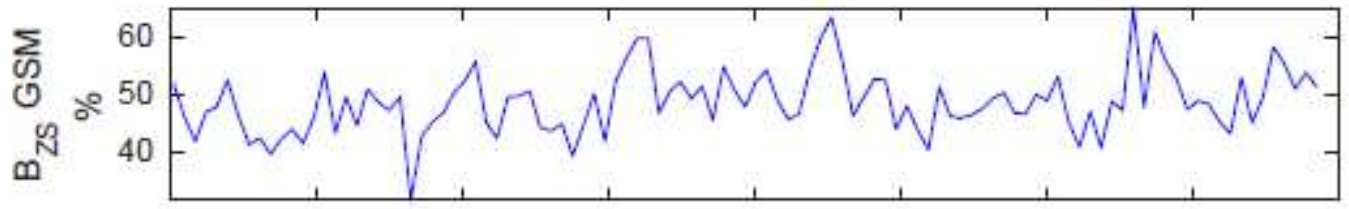

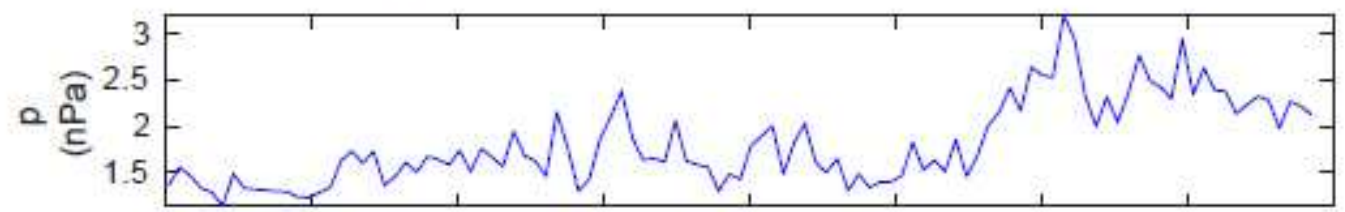

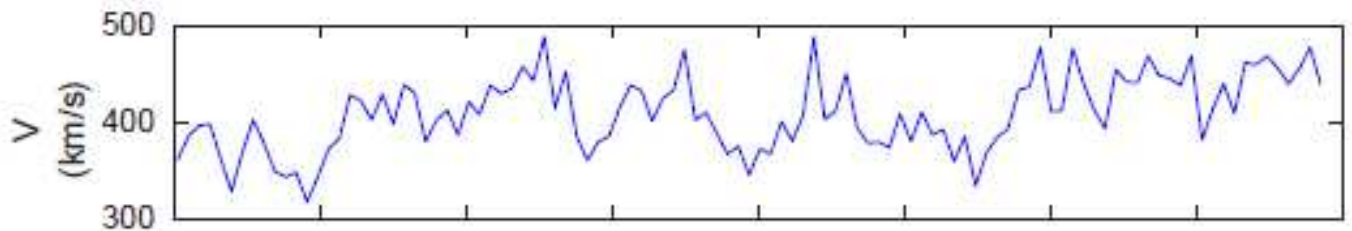

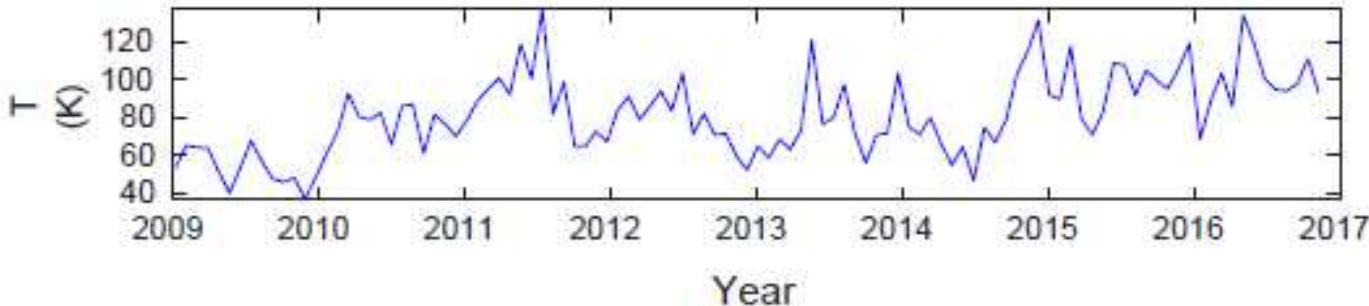

Figure 2

Time series of 27-day-averaged interplanetary magnetic field components and solar wind parameters (IMF and SWP). 


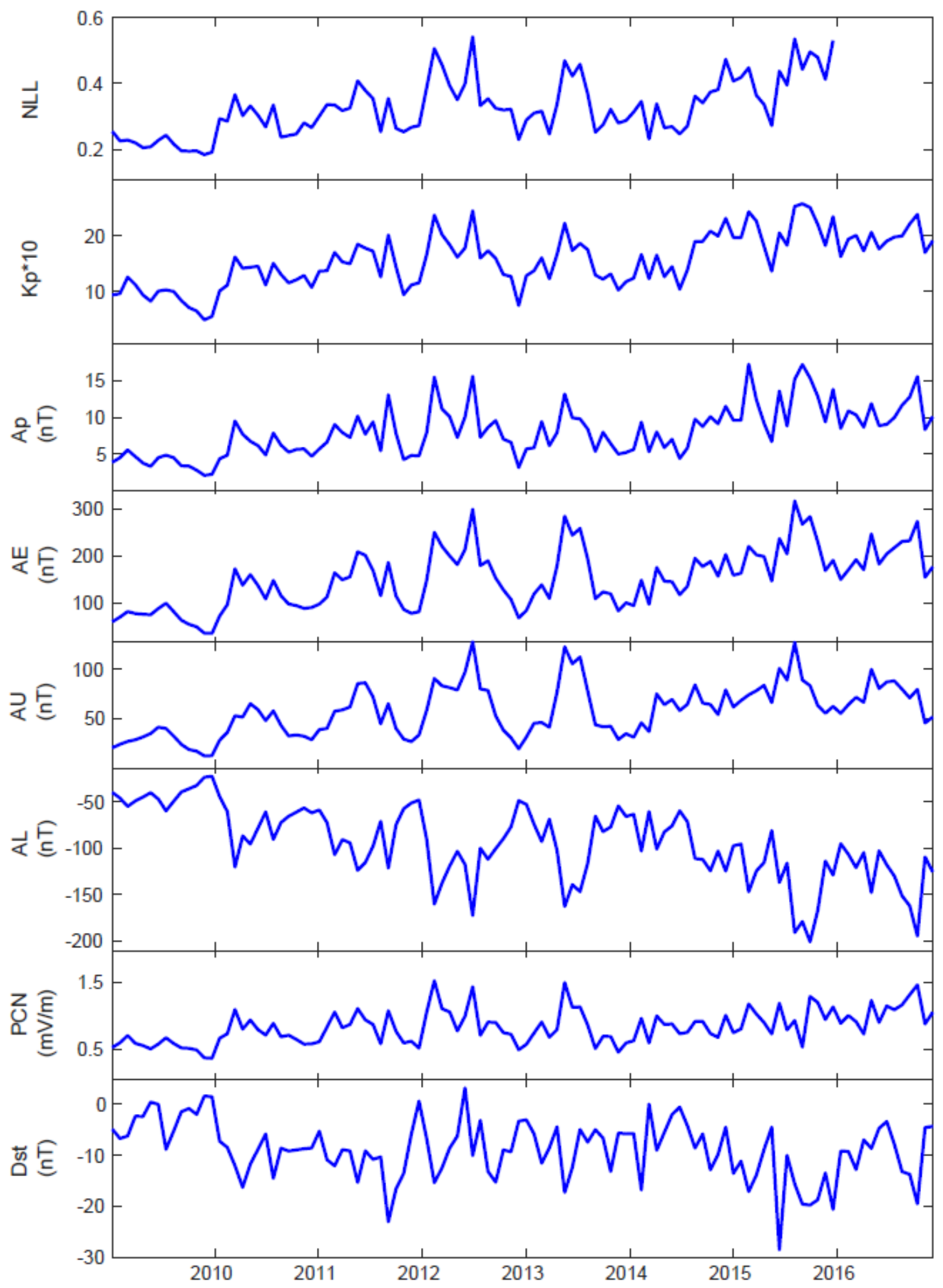

Figure 3

Time series of 27-day-averaged geomagnetic activity indices (GAls) and coupling function NLL. 


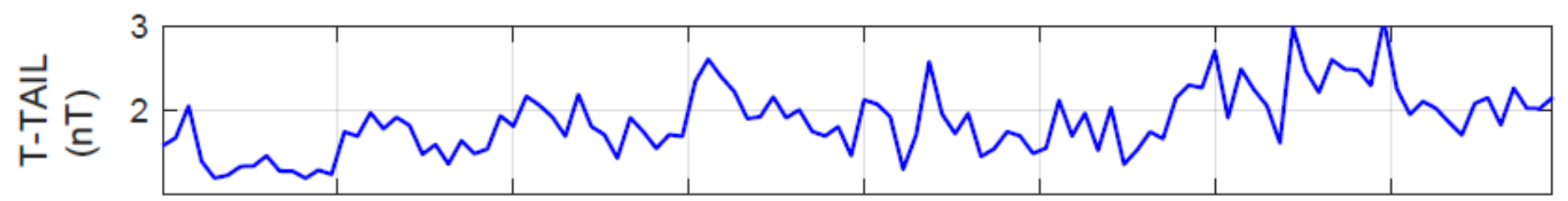
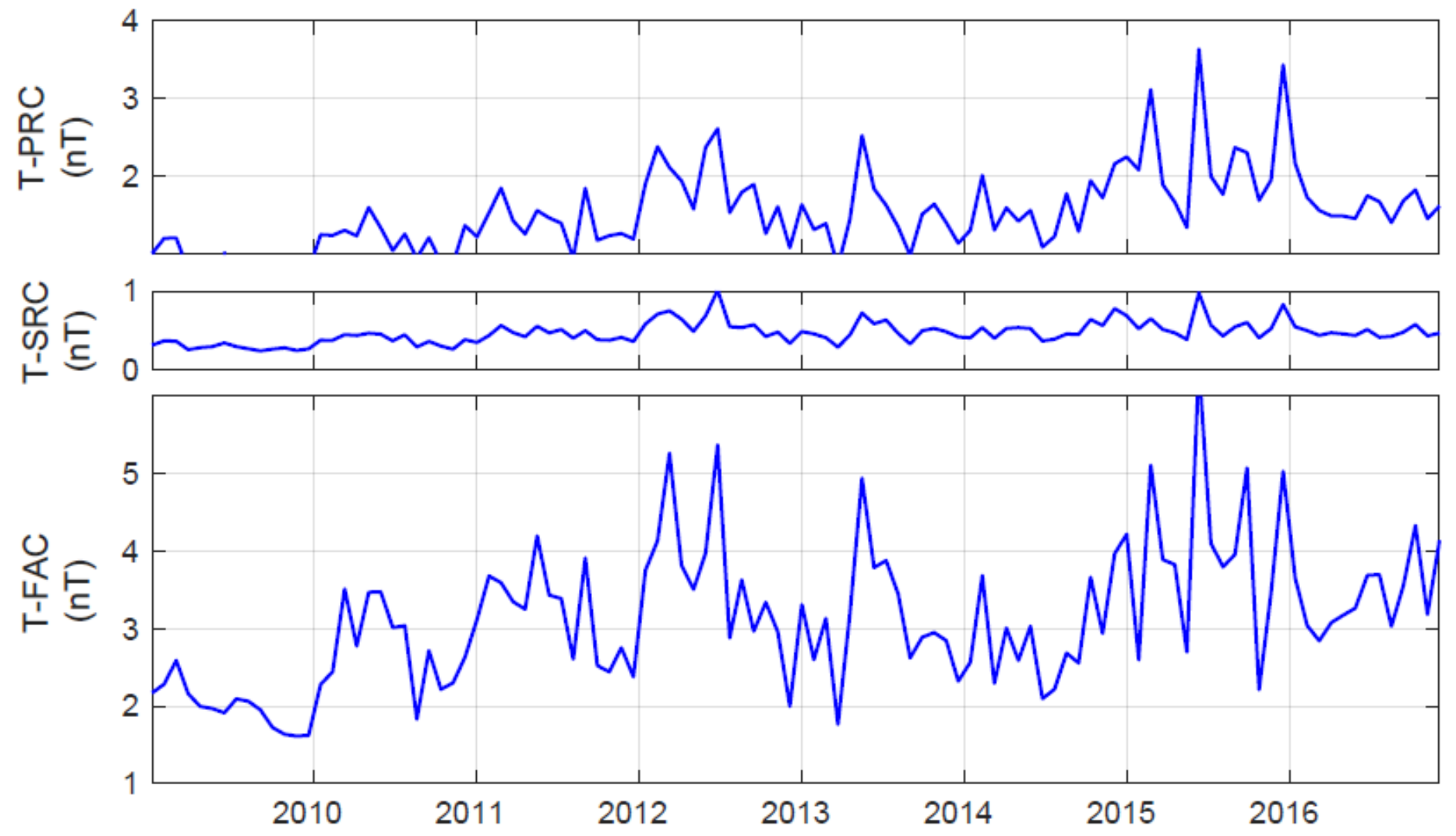

Figure 4

Time series of 27-day-averaged TI magnetospheric indices. 


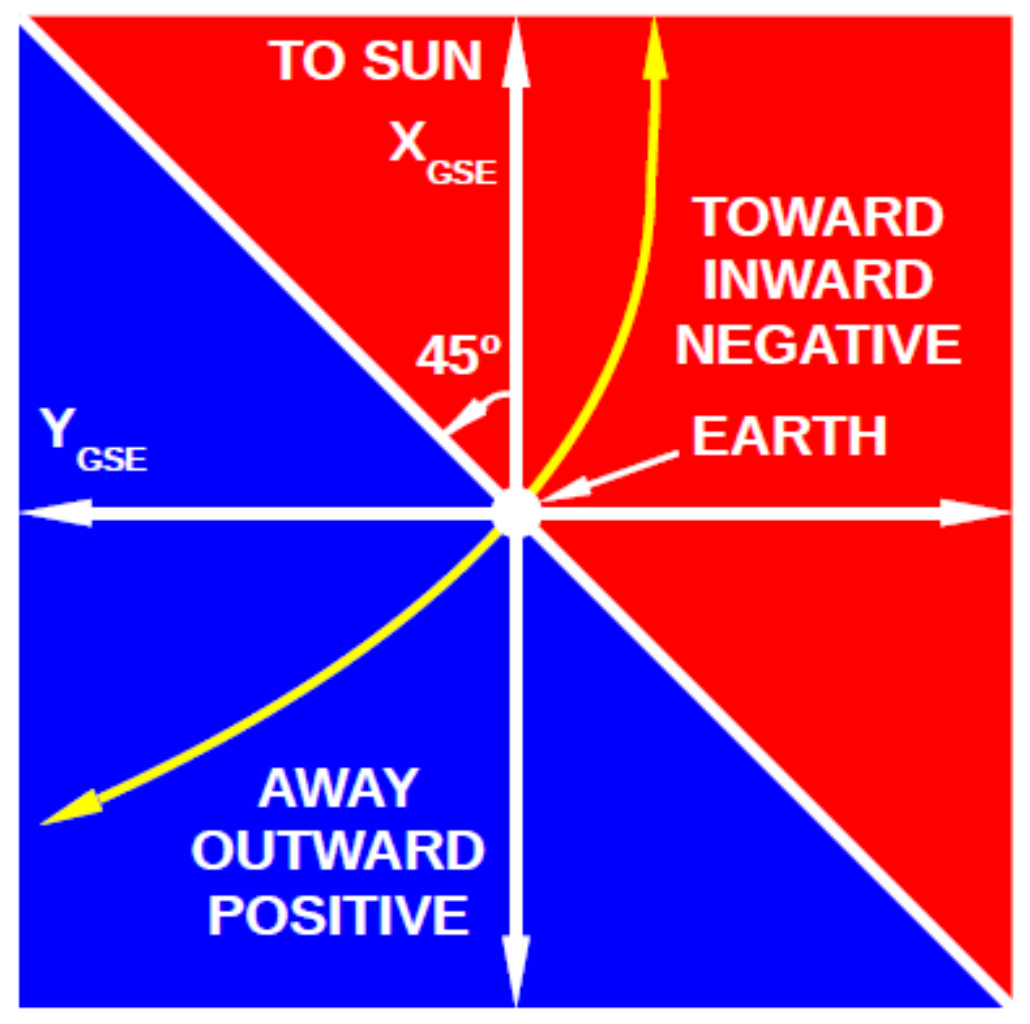

\section{Figure 5}

Definition of away and toward polarity seen from the Earth. Angles in [45o; 225o] are considered away (outward) or positive polarity. Other angles are considered toward (inward) or negative polarity (Sabbah, 1995). Yellow arrows represent the IMF's trajectory and direction in the azimuthal GSE framework. 


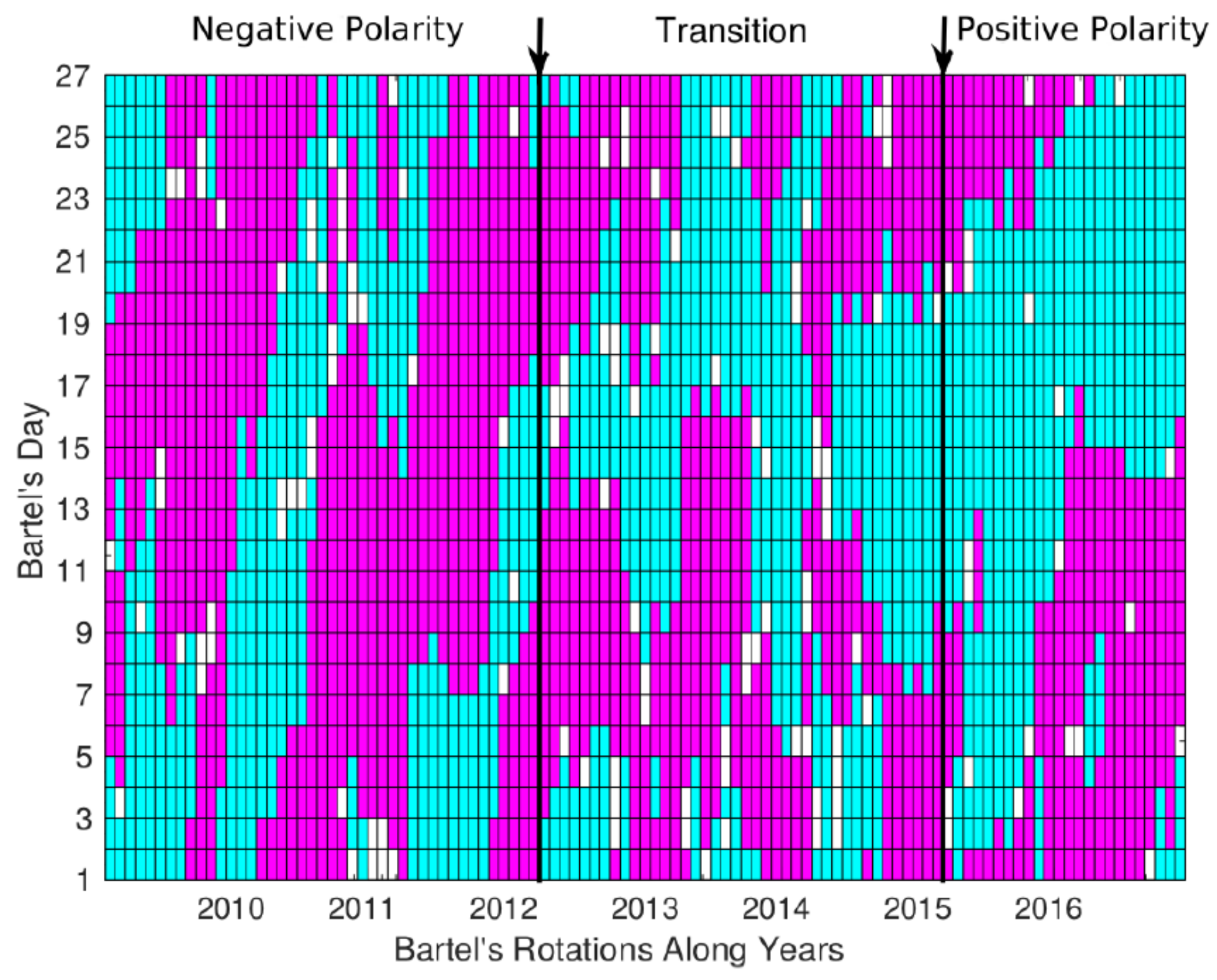

Figure 6

Pixel plot of days with toward/negative polarity (pink) and away/positive polarity (cyan) IMF. White days means missing data or polarity that could not be determined. Each column is a solar rotation (27 days) in the period $2009\{2016$. The solar magnetic eld has negative polarity in $2009\{2012$ and has positive polarity in $2015\{2016$. The interval 2012 \{ 2015 is a transition period. 

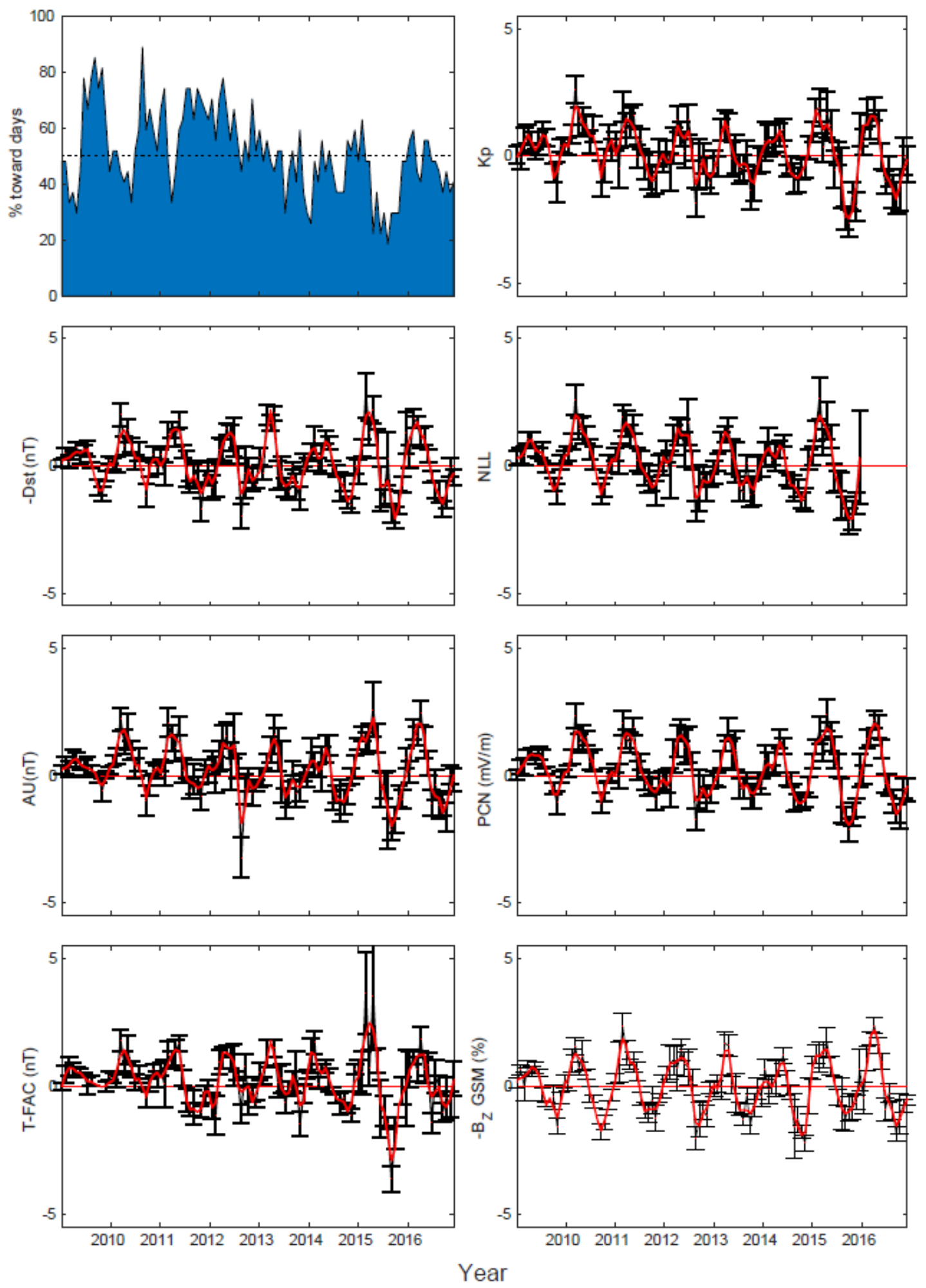

Figure 7

Top-left: Percentage of days with toward IMF during each Bartel's rotation for the whole $2009\{2016$ period. Top-right: Time series of the asymmetry A for the Kp index. From top to bottom, left to right: time series of $A$ for the - Dst, the coupling function NLL, AU, PCN, T-FAC and -BZ GSM, with corresponding error bars. The smooth red curves represent a LOESS regression model, using span=0.5. 


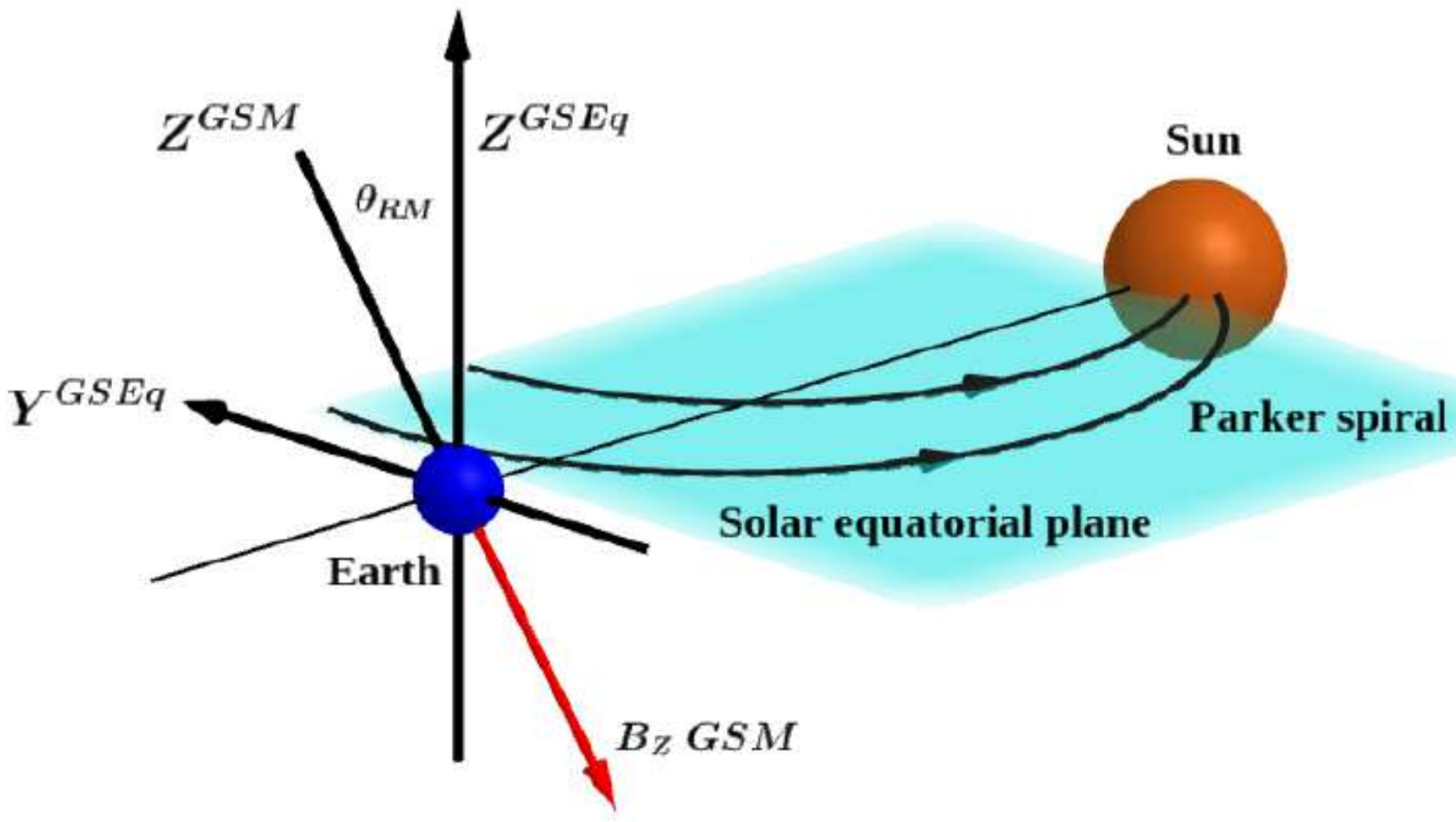

\section{Figure 8}

The $\theta R M$ during the spring equinox, with IMF toward the Sun. The BZ GSM southward (red vector) increases due to the contribution of the negative BY GSEq. Figure adapted from Poblet, Azpilicueta, and Lam (2020)

\section{Supplementary Files}

This is a list of supplementary files associated with this preprint. Click to download.

- BoundaryList20092016.txt

- TowardAwayWilcoxYC20092016.csv 